\title{
Articulo Original / Original Article \\ Medicinal plants and their popular use in Boa Esperança Settlement, Piracanjuba, Goiás, Brazil
}

[Plantas medicinales y su uso popular en el asentamiento de Boa Esperança, Piracanjuba, Goiás, Brasil]

\author{
Brenda Oliveira Guimarães ${ }^{1}$, Isa Lucia de Morais $^{1} \&$ Ana Paula de Oliveira ${ }^{2}$ \\ ${ }^{1}$ State University of Goiás, UEG, Brazil \\ ${ }^{2}$ Institute of Socioenvironmental Studies, Federal University of Goiás, UFG, Brazil
}

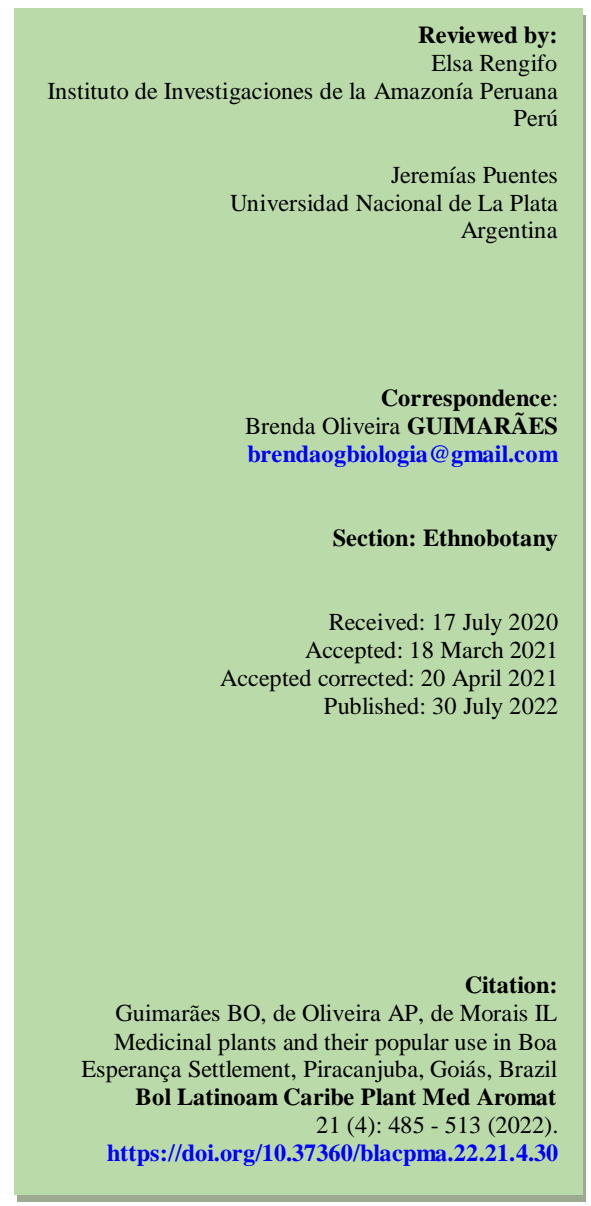

Abstract: The present study showed medicinal plants and their popular use in the rural settlement Boa Esperança, in Piracanjuba, GO, Brazil. Data were collected from November 2017 to February 2018. A total of 176 species (mostly native) were sampled with the predominance of use to treat respiratory, gastrointestinal problems, kidney stones and various types of infections. The species with the highest number of informations were Dilodendron bipinnatum Radlk.; Morus sp.; Pterodon emarginatus Vogel; Qualea grandiflora Mart. and Cochlospermum regium (Mart. ex Schrank) Pilg. With the exception of $C$. regium, the others species had the maximum use agreement value. The study showed a high richness of species used by settlers. The dissemination of this knowledge of medicinal plants, with an emphasis on native plants, can assist in strengthening the cultural knowledge of the local community, encouraging the environmental conservation of various medicinal species used in the region.

Keywords: Cerrado; Tradicional knowledge; Ethnobotany; Popular medicine; Agrarian reform.

Resumen: El presente estudio mostró plantas medicinales y su uso popular en el asentamiento rural Boa Esperança, en Piracanjuba, GO, Brasil. Los datos se recolectaron desde noviembre de 2017 hasta febrero de 2018. Se muestrearon un total de 176 especies (en su mayoría nativas) con predominio de uso para tratar problemas respiratorios, gastrointestinales, cálculos renales y diversos tipos de infecciones. Las especies con mayor número de información fueron Dilodendron bipinnatum Radlk.; Morus sp.; Pterodon emarginatus Vogel; Qualea grandiflora Mart. y Cochlospermum regium (Mart. ex Schrank) Pilg. Con la excepción de C. regium, las otras especies tuvieron el valor de acuerdo de uso máximo. El estudio mostró una gran riqueza de especies utilizadas por los colonos. La difusión de este conocimiento de las plantas medicinales, con énfasis en las plantas nativas, puede ayudar a fortalecer el conocimiento cultural de la comunidad local, fomentando la conservación ambiental de diversas especies medicinales utilizadas en la región.

Palabras clave: Cerrado; Conocimiento tradicional; Etnobotánica; Medicina popular; Reforma agraria. 


\section{INTRODUCTION}

The Cerrado is a Brazilian phytogeographic domain of great importance for its extension, ecological diversity, carbon stocks and hydrological function. Added to this is the presence of a sociocultural diversity consisting of indigenous communities, quilombolas and small agroextractivist producers (Silva \& Peixoto, 2013; Camargo et al., 2014; Bicalho \& Miranda, 2015; Dutra \& Souza, 2017; Pitta \& Vega, 2017; Porto-Gonçalves, 2019).

The sociocultural diversity culminates in valuable traditional knowledge especially those inherent to the various uses of the Cerrado flora. This knowledge about the uses of plants is valued by ethnobotanical science and supports consistent arguments for the conservation of the Cerrado in the process of economic development (Silva \& Peixoto, 2013; Camargo et al., 2014; Bicalho \& Miranda, 2015; Dutra \& Souza, 2017; Pitta \& Vega, 2017; Porto-Gonçalves, 2019).

There is still a great lack of ethnobotanical surveys and extractive potential in the cerrado, mainly in rural settlements, although much of the flora in the cerrado is widely explored by popular knowledge.

It is estimated that in the Cerrado there are more than 600 medicinal plant species, which justifies studies on the medicinal potential of local plants (Guarim Neto \& Morais, 2003). Among these studies, the ethnobotanical inventory in a given locality is anchored and based on knowledge about the management of native species such a medicinal, food, tintorial, timber, textiles, ornamentals, among others. Ethnoknowledge is intrinsic to the experiences of traditional communities whether indigenous, rural or urban, which bring in their bulge great cultural diversity (Almeida et al., 1998).

In this context, medicinal plants continue to occupy a prominent place in the therapeutic arsenal and it is often the only resource of several Brazilian communities (Maciel et al., 2002), especially those located in the rural area, with difficult access to urban areas or where people don't have means of movement. Thus, the present study aimed to sample medicinal plants and their popular use in the rural settlement Boa Esperança, Piracanjuba, GO.

\section{MATERIAL AND METHODS}

\section{Characterization of the study area}

The research was carried out in the Boa
Esperança settlement, located in the municipality of Piracanjuba, Goiás, Brazil (Figure No. 1). The settlement was created on July 1995 and has an area of 1,743.1 hectares. The distance from the settlement to the urban area is about 14 kilometers (17¹8’38.62”'S - 4852'57.31'”W).

The settlement consists of 50 families. Each family legally settled in the communities by INCRA (National Institute of Colonization and Agrarian Reform) received a plot of approximately 5 hectares (INCRA, 2017). The plots, as lots are called, are used for agriculture under family economy. Cattle raising is the main economic activity based on milk production. Agriculture is subsistence with the planting of corn, tobacco, cassava and vegetables.

The predominant vegetation around the settlement is dry forest, mainly in the legal reserve of the rural property. There is occurrence of deciduous dry forest at the top of the hill with predominance of rocky outcrop and, at the base, semi-deciduous dry forest. The water courses present gallery forest (at the headwaters) and riparian forest. There are also small fragments of savanna vegetation.

\section{Survey of floristic and ethnobotanical data}

The present study has a record in the National System of Management of Genetic Heritage and Associated Traditional Knowledge (SisGen) according to Law 13,123 of 05/20/2015 (Brazil, 2015), which revoked Provisional Measure 2,186 of 08/23/2001 (Brazil, 2001) under the number A5E365D. Item $V$ of the sole paragraph of Resolution 510 of April 7, 2016 (CNS, 2016) establishes that there will be no record or evaluated by the CEP/CONEP system (Research Ethics Committees/National Research Ethics Committee) research with databases without possibility of individual identification. Because we understand that the data of the present study is within this item, there was no submission of the study in the Committee of Ethics in Research with Human Beings.

Data collection started through an informal conversation with the president of the Boa Esperança Settlement Association with information about the objective and relevance of the research. We present to each participant the importance and objectives of the research and the guarantee of confidentiality as an ethical premise between researcher and interviewees. The consent of the participants was formalized through a Consent Form, as required by Resolution 
Number 466, of December 12, 2012, of the National 2012). Health Council of the Ministry of Health (CNS,
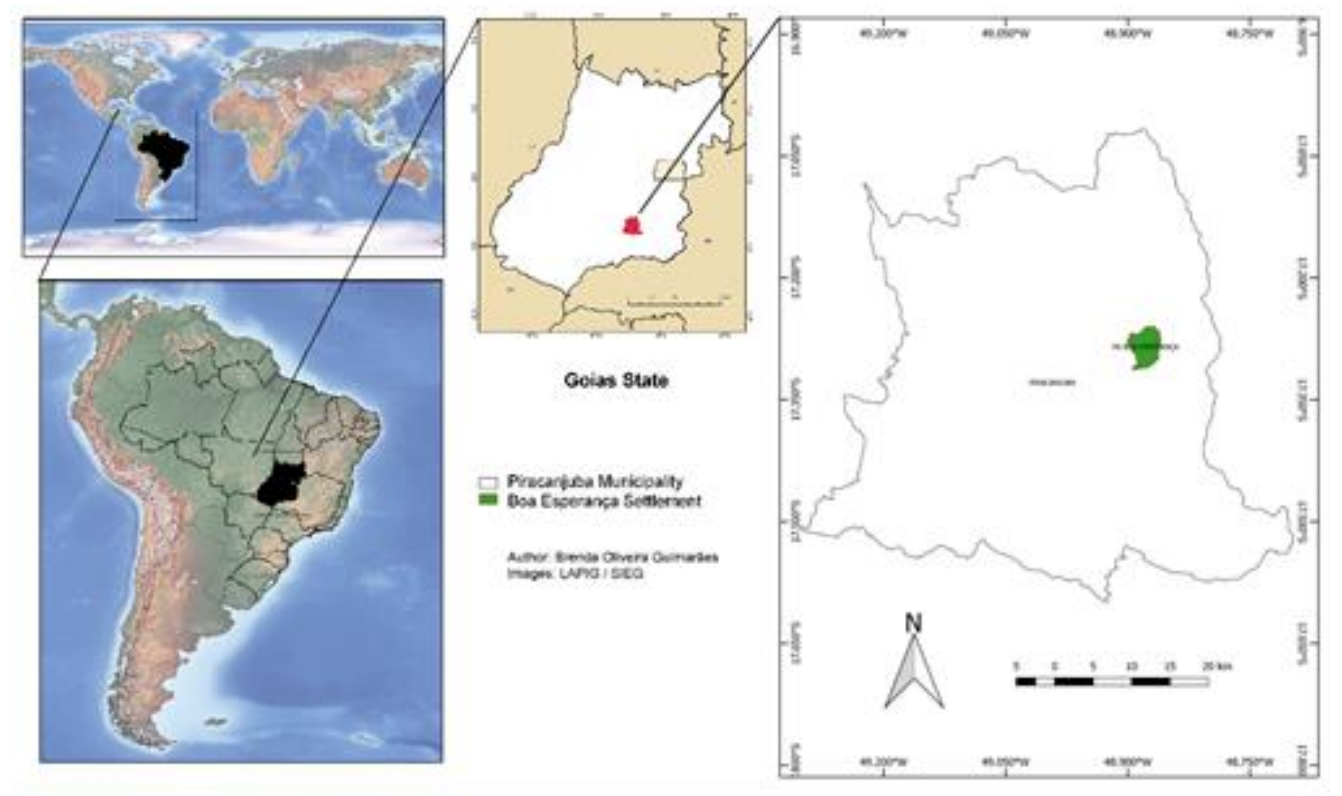

Figure No 1

\section{Map of the study area: Boa Esperança Settlement in Piracanjuba, Goiás, Brazil}

The selection of the target audience was based on information provided by the local community about which people had knowledge about the use of medicinal plants. After the first indication, one person indicated another, and thus successively, until exhausting the number of people in the settlement with ethnobotanical knowledge. This technique is called "snowball" (Albuquerque, 2009). Twelve people belonging to ten families of the settlers were selected. Data collection consisted of semi-structured interviews using a questionnaire containing 21 questions (adapted from Martin (1995)). During the interviews, written and recorded records were performed to ensure the maintenance of reliable information to the reports.

The plants mentioned and found in the plots of the interviewees or in nearby locations were photographed and collected. At that moment, data on habit, collection site and morphological characteristics of the specimens were recorded. These collections were followed by each interviewee. The collected material was herborized and incorporated into the collection of Herbarium José Ângelo Rizzo (JAR), from the State University of Goiás (UEG), Southwest Campus - Quirinópolis.
Native plants were classified according to threat status (IUCN, 2017); whether it is the first occurrence for the state of Goiás, endemic or not of Brazil and its distribution in the Brazilian phytogeographic domains according to the Flora do Brasil 2020 website (2018). Exotic plants were classified according to origin according to data provided by the Missouri Botanical Garden (MOBOT, 2018).

Based on the data from the questionnaires, the plants were organized into eleven categories of medicinal uses: DT $=$ diseases associated with the digestive tract; $\mathrm{RS}=$ diseases associated with the respiratory system; DP = diseases associated with dermatological problems; IP $=$ diseases associated with inflammation and pain; NS = diseases associated with the nervous system; CS = diseases associated with the cardiovascular system; UP $=$ diseases associated with urological problems; $\mathrm{PD}=$ parasitic diseases; $\mathrm{GD}=$ gynecological diseases; $\mathrm{OP}=$ orthopedic problems; and SGS = symptoms and general signs, those with symptoms of various nonspecific diseases and or of cultural origin (Amorozo 2002; Medeiros et al., 2004; Silva et al., 2010; Guimarães et al., 2019). 
To verify the relative importance of the plants used in the community in terms of the number of informants and the agreement of the mentioned uses, the plants mentioned by three or more informants were listed. For the analysis of relative agreement of the species, the percentage of agreement regarding the main uses (PMU) was calculated using the following formula (Amorozo \& Gély, 1988):

\section{PMU $=$ number of informants who cited main use $\times 100$ number of informants who cited the use of the species}

The Correction Factor (CF) was calculated for each species, based on the frequency of citation of each species in relation to the citation of the most cited species:

$\mathrm{CF}=$ number of informants who cited the species/number of informants who cited the most cited species

Then the corrected PMU (PMUc) was obtained by the formula: $\mathrm{PMUc}=\mathrm{PMU} \times \mathrm{CF}$.

The high degree of agreement (one that has several informants indicating the same therapeutic purpose) may be indicative of the effectiveness in the treatment of the disease (Friedman et al., 1986). Thus, the greater the agreement, the greater the chances that the mentioned plant contains some chemical compound that validates its use (Pilla et al., 2006). This information supports the selection of plant species for pharmacological tests in order to prove the efficacy of active ingredients in the treatment of a given disease (Pinto et al., 2006).

To evaluate the diversity of sampled plants, the Shannon-Wiener diversity index was performed $\left(\mathrm{H}^{\prime}\right)$ :

$\mathrm{H}^{\prime}=-\sum$ (pi) (ln pi), where $\mathrm{H}^{\prime}=$ ShannonWiener diversity index, $\mathrm{pi}=\mathrm{ni} / \mathrm{N}$, where $\mathrm{ni}=$ number of citations per species and $\mathrm{N}=$ total number of citations (Magurran, 1988).

The Pielou Equability Index is derived from the Shannon-Wiener diversity index and expresses the uniform distribution of individuals among existing species (Pielou, 1966). This index has a range from 0 (minimum uniformity) to 1 (maximum uniformity). The Pielou equitability index or uniformity was obtained using the formula:

$\mathrm{e}=\mathrm{H}^{\prime} / \log \mathrm{S}$, where $\mathrm{e}=$ Pielou equitability index or uniformity; $\mathrm{H}^{\prime}=$ Shannon-Wiener diversity index and $\mathrm{S}=$ richness or number of species present in the community (Krebs, 1989).
Floristic similarity (comparing studies conducted with other communities and rural settlements in the Cerrado) was investigated using the Jaccard similarity index. The clustering dendogram based on the Jaccard coefficients was made using the UPGMA (Unweighted Pair Group Method with Arithmetic Mean) with the PAST program.

\section{RESULTS AND DISCUSSION Interviewee profile}

Twelve interviews were carried out in 10 families, representing $20 \%$ of the number of families in the settlement. The interviewees were nine women and three men. The greatest female representation was also registered in the study by Silva et al. (2010) on the use of medicinal plants in rural communities in the southwestern region of Goiás. Women, in addition to taking care of their children, among other relatives when they are sick, also take care of food crops, handicrafts, and ornaments made with local plants (Pasa et al., 2015). In the case of the interviewees, in addition to taking care of the cultivated plants, they constantly attend the nearby Cerrado vegetation types.

Research participants are aged between 26 and 69 years. The two most frequent age groups, in an equal number of respondents, were 51 to 60 and 61 to 70 years old $(41.67 \%, n=5$, each). Other studies showed a greater number of people in the age group above 60 years (Mota et al., 2015; Duarte \& Pasa, 2016; Brito et al., 2017; Guimarães et al., 2019). In Brazil, most young people are not interested in treatment with medicinal plants, and for this reason, they are unaware of them (Oliveira \& Menini Neto, 2012). This has culminated in the loss of ethnobotanical knowledge over the generations (Löbler et al., 2014).

As for the naturalness of the participants, two 
are from Bahia, one from Tocantins, one from Minas Gerais and eight are from Goiás (of these two are from Piracanjuba). All participants are of rural origin, with the majority living in the settlement since its implementation $(66.67 \%)$. The education level of the majority of the interviewees is, in equal numbers, complete high school and incomplete elementary school $(33.33 \%, \mathrm{n}=4$, each), followed by illiterate $(16.67 \%, \mathrm{n}=2)$ and incomplete high school and complete elementary school $(8.33 \%, \mathrm{n}=1$, each). This result differs from the results of most ethnobotanical studies in the country, in which most respondents are illiterate, in equal numbers, complete high school and incomplete elementary school $(33.33 \%, \mathrm{n}=4$, each), followed by illiterates $(16.67 \%, \mathrm{n}=2)$ and education incomplete high school and complete elementary school $(8.33 \%, \mathrm{n}=1$, each). This result differs from the results of most ethnobotanical studies in the country, in which a large part of the interviewees is illiterate (Cunha \& Bortolotto, 2011; Oliveira \& Menini Neto, 2012; Löbler et al., 2014; Mota et al., 2015; Pasa et al., 2015).

The knowledge about medicinal plants acquired by the target audience was diversified, with different contributions for the same interviewee. The majority affirmed, in equal numbers, having obtained this knowledge through their parents and participating in courses offered by entities that work in the settlements, such as INCRA and SENAR (33.33\%, $\mathrm{n}=7$, each); followed by the teachings of grandparents $(19.05 \%, \mathrm{n}=4)$; and, to a lesser extent, through friends and neighbors $(9.52 \%, \mathrm{n}=2)$. According to them, most of the interviewees are regularly sharing plant seedlings and medicinal recipes. In this context, traditional knowledge is, most of the time, produced in social relations and transmitted and disseminated orally (Dias \& Laureano, 2009).

\section{Ethnobotanical data}

A total of 176 medicinal plant species were recorded (Figure No. 2), distributed in 71 families and 155 genera (Table No. 1). Fabaceae (16 species), Asteraceae (15) and Lamiaceae (13) were the families with the highest species richness. These families were also the richest in other ethnobotanical studies (Cunha \& Bortolotto, 2011; Rodrigues \& Andrade, 2014; Costa \& Marinho, 2016; Gois et al., 2016).

The richest genera in species were Allium L.,
Citrus L. and Mentha L., all with four species each. In other ethnobotanical studies, in a Cerrado area, similar results were found (Amorozo, 2002; Borba \& Macedo, 2006; Silva \& Proença, 2007; Alves \& Povh, 2013; Ferreira et al., 2015; Guimarães et al., 2019).

Among the medicinal species mentioned, most are native $(55,11 \%, \mathrm{n}=97)$, followed by exotics $(28,40 \%, \mathrm{n}=50)$ and naturalized $(16.47 \%, \mathrm{n}=29)$ (Table No. 1). Among the native ones eight are endemic to Brazil: Adenocalymma nodosum, Cayaponia tayuya, Dorstenia cayapia, Jacaranda rufa, Smilax brasiliensis, Solanum cernuum, Vochysia elliptica and Zeyheria montana. The use of medicinal species, the majority of which are native, has also been recorded by other ethnobotanical research (Oliveira et al., 2010; Cunha \& Bortolotto, 2011; Leandro et al., 2017; Guimarães et al., 2019). There was a predominance of herbaceous habit (72), followed by arboreal (59), shrub (55), sub-shrub (23) and liana (14). A similar result was found in the ethnobotanical study with medicinal plants carried out in rural settlements in southwest Goiás (Silva et al., 2010).

Most of the 93 native species $(91.76 \%)$ are found in the Cerrado phytogeographic domain. However, these species are not exclusive to this domain and they are shared mainly with the Atlantic Forest (74.12\%), Amazon (67.06\%) and Caatinga (65.88\%) (Figure No. 3). Among these species, four are exclusive to a Brazilian phytogeographic domain, being Aristolochia esperanzae, Gomphrena arborescens and Lafoensia pacari for the Cerrado; Sambucus australis and Myrocarpus frondosus for the Atlantic Forest, and Myroxylon balsamum for the Amazon.

Regarding threat status (Flora do Brasil, 2020), most of the native medicinal species mentioned were not evaluated $(40 \%, n=39)$ (Table No. 1). Therefore, it is important to expand the studies to evaluate the threat status of these species to establish future conservation strategies. For Baccharis crispa, Cecropia pachystachya and Vochysia elliptica this evaluation is ongoing. For Xylopia aromatica, Zeyheria montana, Maytenus ilicifolia, Mikania glomerata, Myracrodruon urundeuva, Dioscorea trifida, Dipteryx alata, Stryphnodendron adstringens, Hymenaea courbaril, Genipa americana, Dilodendron bipinnatum and Terminalia argentea status is least concern (LC),

\section{Boletín Latinoamericano y del Caribe de Plantas Medicinales y Aromáticas / 489}


while for Lychnophora ericoides, Amburana cearenses and Handroanthus impetiginosus the status is near threated (NT), requiring studies to prevent possible risks of extinction. The species Anemopaegma arvense is in the threat category as endangered (EN), being at a very high risk of extinction of nature. This species was found at the study site in the reproductive period, the which demonstrates the importance of ethnobotanical studies for the conservation of plant species.

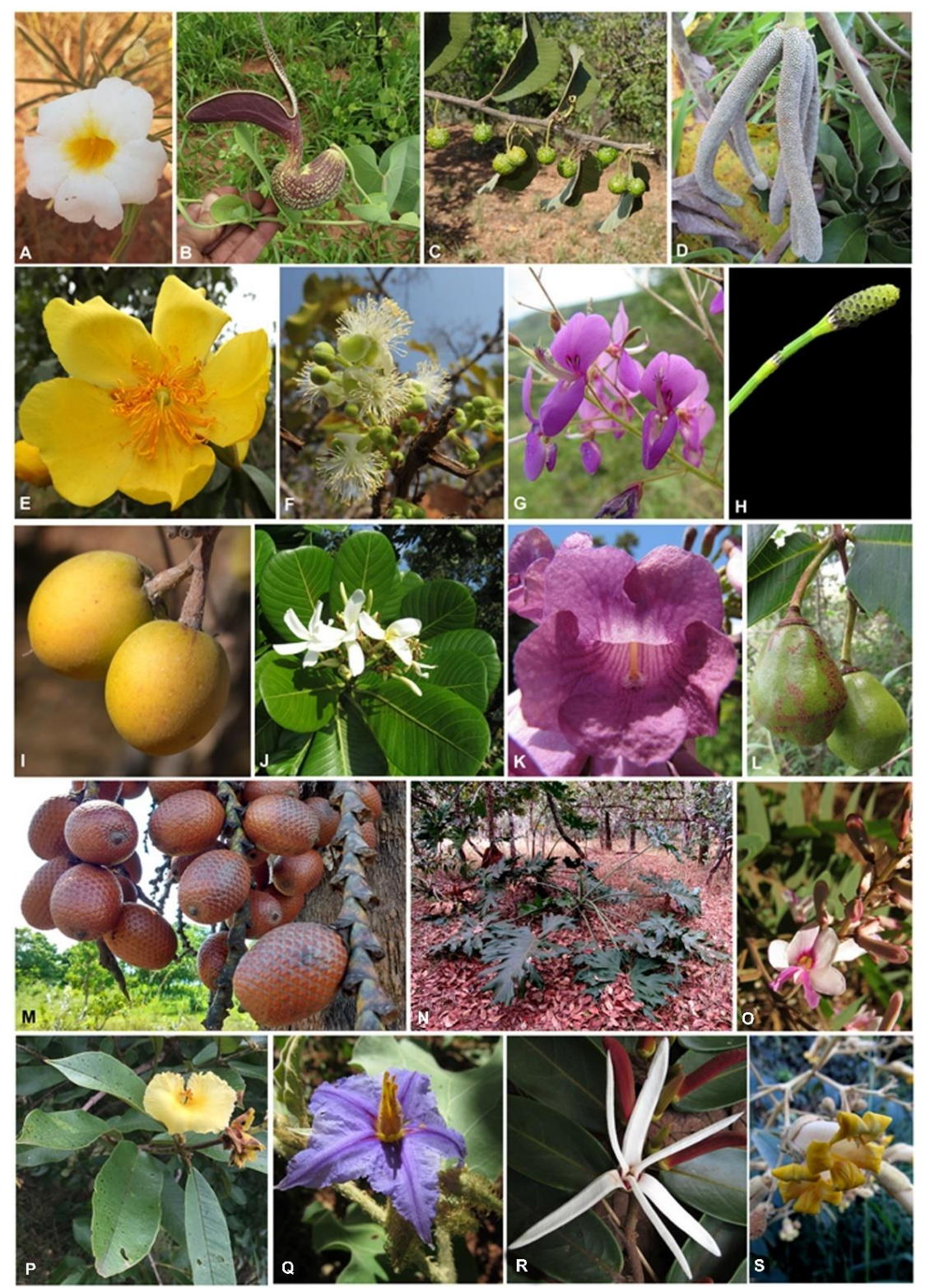

Figure No 2

Some of the medicinal species sampled in the study area Boa Esperança Settlement in Piracanjuba, Goiás, Brazil. A) Anemopagma arvense; B) Aristolochia esperanzae; C) Brosimum gaudichaudii; D) Cecropia pachystachya; E) Cochlospermum regium; F) Curatella americana; G) Desmodium incanum; H) Equisetum giganteum; I) Hancornia speciosa; J) Himatanthus obovatus; K) Jacaranda rufa; L) Lafoensia pacari; M) Mauritia flexuosa; N) Philodendron bipinnatifidum; O) Pterodon emarginatus; P) Qualea grandiflora; Q) Solanum lycocarpum; R) Xylopia aromatica; S) Zeyheria montana. 
Table No. 1

List of medicinal plants used in the Boa Esperança rural settlement of Piracanjuba, GO. Habit: $\mathbf{H}=$ herbaceous; $\mathbf{A r}=$ arboreal; $\mathbf{S}=$ shrub; Sb = sub-shrub; L = liana. IUCN (International Union for Conservation of Nature) for native species: $\mathrm{NE}=$ Not evaluated, NT $=$ Near threatened, EN = Endangered, $\mathbf{L C}=$ Least concern

\begin{tabular}{|c|c|c|c|c|}
\hline Family & Species & Habit & Source & $\begin{array}{l}\text { IUCN/Endemic to Brazil/ } \\
\text { Phytogeographic domains }\end{array}$ \\
\hline Acanthaceae & Justicia pectoralis Jacq. & $\mathrm{H}$ & native & NE/no/Amazon, Cerrado \\
\hline Adoxaceae & $\begin{array}{l}\text { Sambucus australis Cham. } \\
\text { \& Schltdl. }\end{array}$ & $\mathrm{S}, \mathrm{Ar}$ & native & NE/no/Atlantic Forest \\
\hline Alismataceae & $\begin{array}{l}\text { Echinodorus grandiflorus } \\
\text { (Cham. \& Schltr.) Micheli }\end{array}$ & $\mathrm{H}$ & native & $\begin{array}{c}\text { NE/no/Caatinga, Cerrado, Atlantic } \\
\text { Forest }\end{array}$ \\
\hline \multirow{3}{*}{ Amaranthaceae } & $\begin{array}{c}\text { Alternanthera brasiliana } \\
\text { (L.) O. Kunt }\end{array}$ & $\mathrm{H}, \mathrm{Sb}$ & exotic (Egypt) & ( \\
\hline & $\begin{array}{l}\text { Dysphania ambrosioides } \\
\text { (L.) Mosyakin \& Clemants }\end{array}$ & $\mathrm{Sb}$ & $\begin{array}{l}\text { naturalized } \\
\text { (Colombia) }\end{array}$ & - \\
\hline & $\begin{array}{c}\text { Gomphrena arborescens } \\
\text { L.f. }\end{array}$ & $\mathrm{Sb}$ & native & $\mathrm{NE} /$ no/Cerrado \\
\hline \multirow{4}{*}{ Amaryllidaceae } & Allium cepa $\mathrm{L}$. & $\mathrm{H}$ & exotic (Asia) & - \\
\hline & Allium fistulosum $\mathrm{L}$. & $\mathrm{H}$ & exotic (Asia) & - \\
\hline & Allium porrum L. & $\mathrm{H}$ & exotic (Asia) & - \\
\hline & Allium sativum L. & $\mathrm{H}$ & $\begin{array}{l}\text { exotic (Middle } \\
\text { East) }\end{array}$ & - \\
\hline \multirow[b]{2}{*}{ Anacardiaceae } & Mangifera indica L. & $\mathrm{Ar}$ & exotic (India) & - \\
\hline & $\begin{array}{l}\text { Myracrodruon urundeuva } \\
\text { Allemão. }\end{array}$ & $\mathrm{S}$ & native & $\begin{array}{c}\text { LC/no/Caatinga, Cerrado, } \\
\text { Atlantic Forest } \\
\end{array}$ \\
\hline \multirow{2}{*}{ Annonaceae } & Annona muricata $\mathrm{L}$. & $\mathrm{Ar}$ & $\begin{array}{c}\text { naturalized } \\
\text { (Central America) }\end{array}$ & 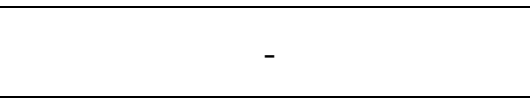 \\
\hline & $\begin{array}{c}\text { Xylopia aromatica (Lam.) } \\
\text { Mart. }\end{array}$ & $\mathrm{S}, \mathrm{Ar}$ & native & LC/no/Amazon, Cerrado \\
\hline \multirow{5}{*}{ Apiaceae } & Eryngium foetidum $\mathrm{L}$. & $\mathrm{H}$ & native & $\begin{array}{c}\text { NE/no/Amazon, Caatinga, } \\
\text { Cerrado, Atlantic Forest, Pampa, } \\
\text { Pantanal }\end{array}$ \\
\hline & Cuminum cyminum $\mathrm{L}$. & $\mathrm{H}$ & $\begin{array}{c}\text { exotic } \\
\text { (Mediterranean) }\end{array}$ & 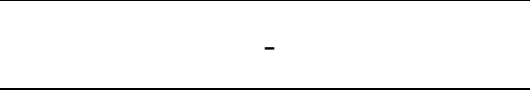 \\
\hline & Daucus carota $\mathrm{L}$. & $\mathrm{H}$ & $\begin{array}{c}\text { naturalized } \\
\text { (Europe, Asia) } \\
\end{array}$ & - \\
\hline & Foeniculum vulgare Mill. & $\mathrm{H}$ & $\begin{array}{c}\text { naturalized } \\
\text { (Mediterranean) }\end{array}$ & - \\
\hline & $\begin{array}{c}\text { Petroselinum crispum } \\
\text { (Mill.) Fuss }\end{array}$ & $\mathrm{H}$ & $\begin{array}{l}\text { exotic (likely } \\
\text { Europe) }\end{array}$ & - \\
\hline \multirow{4}{*}{ Apocynaceae } & $\begin{array}{c}\text { Catharanthus roseus (L.) } \\
\text { Don }\end{array}$ & $\mathrm{H}$ & $\begin{array}{c}\text { naturalized } \\
\text { (Madagascar) }\end{array}$ & - \\
\hline & Hancornia speciosa Gomes & Ar & native & $\begin{array}{c}\text { NE/no/Amazon, Caatinga, } \\
\text { Cerrado, Atlantic Forest }\end{array}$ \\
\hline & $\begin{array}{l}\text { Himatanthus obovatus } \\
\text { (Müll. Arg.) Woodson }\end{array}$ & $\mathrm{Ar}$ & native & $\begin{array}{c}\text { NE/no/Amazon, Caatinga, } \\
\text { Cerrado }\end{array}$ \\
\hline & $\begin{array}{l}\text { Mandevilla velame (A.St.- } \\
\text { Hil.) Pichon }\end{array}$ & $\mathrm{Sb}$ & native & NE/no/Cerrado, Pampa \\
\hline
\end{tabular}

Boletín Latinoamericano y del Caribe de Plantas Medicinales y Aromáticas / 491 


\begin{tabular}{|c|c|c|c|c|}
\hline & $\begin{array}{l}\text { Peltastes peltatus (Vell.) } \\
\text { Woodson }\end{array}$ & $\mathrm{L}$ & naturalized & NE/no/Cerrado, Atlantic Forest \\
\hline & $\begin{array}{l}\text { Thevetia peruviana (Pers.) } \\
\text { K. Schum }\end{array}$ & Ar, S & native & NE/no/Amazon, Cerrado \\
\hline \multirow{2}{*}{ Araceae } & $\begin{array}{c}\text { Philodendron } \\
\text { bipinnatifidum Schott } \\
\end{array}$ & $\mathrm{H}$ & native & NE/no/Cerrado, Atlantic Forest \\
\hline & $\begin{array}{l}\text { Xanthosoma sagittifolium } \\
\text { (L.) Schott }\end{array}$ & $\mathrm{H}$ & $\begin{array}{c}\text { naturalized } \\
\text { (America) }\end{array}$ & 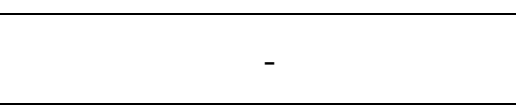 \\
\hline Arecaceae & Mauritia flexuosa L.f. & $\mathrm{H}$ & native & $\begin{array}{l}\text { NE/no/Amazon, Caatinga, } \\
\text { Cerrado }\end{array}$ \\
\hline \multirow{2}{*}{ Aristolochiaceae } & $\begin{array}{l}\text { Aristolochia esperanzae } \\
\text { Kuntze }\end{array}$ & $\mathrm{L}$ & native & NE/no/Cerrado \\
\hline & Aristolochia trilobata $\mathrm{L}$. & $\mathrm{L}$ & native & $\begin{array}{c}\text { NE/no/Amazon, Caatinga, } \\
\text { Atlantic Forest }\end{array}$ \\
\hline Asphodelaceae & Aloe vera (L.) Burm. f. & $\mathrm{H}$ & exotic (Africa) & - \\
\hline \multirow{15}{*}{ Asteraceae } & Achillea millefolium $\mathrm{L}$. & $\mathrm{H}$ & $\begin{array}{c}\text { exotic (Europe, } \\
\text { Asia) }\end{array}$ & 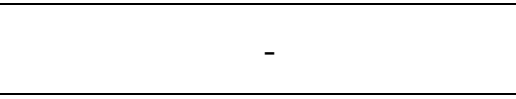 \\
\hline & $\begin{array}{c}\text { Achyrocline satureioides } \\
\text { (Lam.) DC }\end{array}$ & $\mathrm{H}$ & native & $\begin{array}{c}\text { NE/no/Cerrado, Atlantic Forest, } \\
\text { Pampa }\end{array}$ \\
\hline & $\begin{array}{c}\text { Acmella oleraceae (L.) R. } \\
\text { K. Jansen }\end{array}$ & $\mathrm{H}$ & $\begin{array}{c}\text { naturalized (South } \\
\text { America) }\end{array}$ & $e^{2}$ \\
\hline & Ageratum conyzoides $\mathrm{L}$. & $\mathrm{H}, \mathrm{Sb}$ & native & $\begin{array}{c}\text { NE/no/Amazon, Caatinga, } \\
\text { Cerrado, Atlantic Forest, Pampa, } \\
\text { Pantanal }\end{array}$ \\
\hline & Artemisia absinthium L. & $\mathrm{Sb}$ & $\begin{array}{l}\text { exotic (Asia, } \\
\text { Europe) }\end{array}$ & ( \\
\hline & Baccharis crispa Spreng. & $\mathrm{Sb}$ & native & $\begin{array}{c}\text { NE/no/Caatinga, Cerrado, } \\
\text { Atlantic Forest, Pampa }\end{array}$ \\
\hline & Bidens pilosa $\mathrm{L}$. & $\mathrm{H}$ & $\begin{array}{l}\text { naturalized (likely } \\
\text { America) }\end{array}$ & 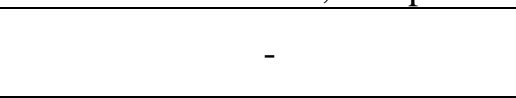 \\
\hline & $\begin{array}{l}\text { Dasyphyllum brasiliense } \\
\text { (Spreng.) Cabrera }\end{array}$ & $\mathrm{S}, \mathrm{Ar}$ & native & NE/no/Cerrado, Atlantic Forest \\
\hline & $\begin{array}{c}\text { Lychnophora ericoides } \\
\text { Mart. }\end{array}$ & $\mathrm{Ar}$ & native & NT/yes/Caatinga, Cerrado \\
\hline & Matricaria recutita $\mathrm{L}$. & $\mathrm{H}$ & $\begin{array}{l}\text { exotic (Europe, } \\
\text { Africa) }\end{array}$ & ( \\
\hline & Mikania glomerata Spreng. & $\mathrm{L}$ & native & LC/ no/Cerrado, Atlantic Forest \\
\hline & Solidago chilensis Meyen & $\mathrm{Sb}$ & native & $\begin{array}{c}\text { NE/no/Caatinga, Cerrado, } \\
\text { Atlantic Forest, Pampa }\end{array}$ \\
\hline & Tagetes patula L. & $\mathrm{H}$ & native & $\mathrm{NE} /$ no/there is no information \\
\hline & $\begin{array}{l}\text { Tanacetum parthenium (L.) } \\
\text { Sch. Bip. }\end{array}$ & $\mathrm{H}$ & exotic (Europe) & e \\
\hline & $\begin{array}{c}\text { Vernonanthura polyanthes } \\
\text { (Sprengel) Vega \& } \\
\text { Dematteis }\end{array}$ & $\mathrm{S}$ & native & $\mathrm{NE} /$ no/there is no information \\
\hline Balsaminaceae & Impatiens balsamina $\mathrm{L}$. & $\mathrm{H}$ & naturalized & NE/no/Atlantic Forest \\
\hline Bignoniaceae & $\begin{array}{l}\text { Adenocalymma nodosum } \\
\text { (Silva Manso) } \\
\text { L.G.Lohmann }\end{array}$ & S & $\begin{array}{c}\text { native and } \\
\text { endemic to Brazil }\end{array}$ & $\begin{array}{c}\text { NE/yes/Caatinga, Cerrado, } \\
\text { Atlantic Forest }\end{array}$ \\
\hline
\end{tabular}

Boletín Latinoamericano y del Caribe de Plantas Medicinales y Aromáticas / 492 


\begin{tabular}{|c|c|c|c|c|}
\hline & $\begin{array}{c}\text { Anemopagma arvense } \\
\text { (Vell.) Stellfeld ex de Souza }\end{array}$ & $S$ & native & $\begin{array}{c}\text { EN/no/Amazon, Cerrado, } \\
\text { Atlantic Forest. }\end{array}$ \\
\hline & $\begin{array}{l}\text { Handroanthus impetiginosus } \\
\text { (Mart. ex DC.) Mattos }\end{array}$ & $\mathrm{Ar}$ & native & $\begin{array}{c}\text { NT/no/Amazon, Caatinga, } \\
\text { Cerrado, Atlantic Forest, Pantanal }\end{array}$ \\
\hline & Jacaranda rufa Silva Manso & $\mathrm{S}$ & $\begin{array}{c}\text { native and } \\
\text { endemic to Brazil }\end{array}$ & NE/yes/Cerrado, Atlantic Forest \\
\hline & Zeyheria montana Mart. & $\mathrm{S}$ & $\begin{array}{c}\text { native and } \\
\text { endemic to Brazil }\end{array}$ & $\begin{array}{c}\text { LC/Yes/Amazon, Caatinga, } \\
\text { Cerrado, Atlantic Forest }\end{array}$ \\
\hline \multirow{2}{*}{ Bixaceae } & Bixa orellana $\mathrm{L}$. & $\mathrm{S}, \mathrm{Ar}$ & native & $\begin{array}{c}\text { NE/no/Amazon, Cerrado, Atlantic } \\
\text { Forest }\end{array}$ \\
\hline & $\begin{array}{l}\text { Cochlospermum regium } \\
\text { (Mart. ex Schrank) Pilg. }\end{array}$ & $\mathrm{S}, \mathrm{Sb}$ & native & $\begin{array}{c}\text { LC/no/Amazon, Caatinga, } \\
\text { Cerrado, Pantanal }\end{array}$ \\
\hline Boraginaceae & Symphytumofficinale L. & $\mathrm{H}$ & $\begin{array}{l}\text { exotic (Europe and } \\
\text { Asia) }\end{array}$ & ( \\
\hline \multirow[b]{2}{*}{ Brassicaceae } & $\begin{array}{c}\text { Brassica sylvestris (L.) } \\
\text { Mill.) }\end{array}$ & $\mathrm{H}$ & exotic (Europe) & - \\
\hline & Eruca sativa Mill. & $\mathrm{H}$ & $\begin{array}{c}\text { exotic } \\
\text { (Mediterranean, } \\
\text { Asia) } \\
\end{array}$ & - \\
\hline Cannabaceae & Celtis iguanea (Jacq.) Sarg. & $\mathrm{S}, \mathrm{Ar}$ & native & $\begin{array}{c}\text { NE/no/Amazon, Caatinga, } \\
\text { Cerrado, Atlantic Forest, Pampa, } \\
\text { Pantanal } \\
\end{array}$ \\
\hline Caricaceae & Carica papaya $\mathrm{L}$. & Ar, S & naturalized (India) & - \\
\hline Celastraceae & $\begin{array}{l}\text { Maytenus ilicifolia (Schrad.) } \\
\text { Planch. }\end{array}$ & $S$ & native & $\begin{array}{c}\text { LC /no/Cerrado, Atlantic Forest, } \\
\text { Pampa }\end{array}$ \\
\hline Chenopodiaceae & Beta vulgaris L. & $\mathrm{H}$ & exotic (Europe) & - \\
\hline Combretaceae & Terminalia argentea Mart. & $\mathrm{S}, \mathrm{Ar}$ & native & $\begin{array}{c}\text { LC/no/Amazon, Caatinga, } \\
\text { Cerrado, Atlantic Forest }\end{array}$ \\
\hline Convolvulaceae & $\begin{array}{c}\text { Operculina } \\
\text { macrocarpa (L.) } \\
\text { Urb. }\end{array}$ & $\mathrm{L}$ & native & $\begin{array}{l}\text { LC/no/Amazon, Caatinga, } \\
\text { Cerrado, Atlantic Forest }\end{array}$ \\
\hline Costaceae & $\begin{array}{c}\text { Costus spiralis (Jacq.) } \\
\text { Roscoe } \\
\end{array}$ & $\mathrm{H}$ & native & $\begin{array}{c}\text { NE/no/Amazon, Caatinga, } \\
\text { Cerrado, Atlantic Forest, Pantanal }\end{array}$ \\
\hline \multirow{3}{*}{ Crassulaceae } & Kalanchoe laetivirens Desc. & $\mathrm{H}$ & exotic (Africa) & - \\
\hline & $\begin{array}{l}\text { Kalanchoe pinnata (Lam.) } \\
\text { Pers. }\end{array}$ & $\mathrm{H}$ & $\begin{array}{l}\text { naturalized (likely } \\
\text { Madagascar) }\end{array}$ & - \\
\hline & Sedum dendroideum DC. & $\mathrm{H}$ & exotic (Africa) & - \\
\hline \multirow{5}{*}{ Cucurbitaceae } & $\begin{array}{l}\text { Cayaponia tayuya (Vell.) } \\
\text { Cogn. }\end{array}$ & $\mathrm{L}$ & $\begin{array}{c}\text { native and } \\
\text { endemic to Brazil }\end{array}$ & $\begin{array}{l}\text { NE/yes/Amazon, Caatinga, } \\
\text { Cerrado, Atlantic Forest }\end{array}$ \\
\hline & Cucurbita pepo L. & $\mathrm{L}$ & exotic (Mexico) & - \\
\hline & Cucumis anguria $\mathrm{L}$. & $\mathrm{L}$ & native & $\begin{array}{c}\text { NE/no/Amazon, Cerrado, } \\
\text { Atlantic Forest }\end{array}$ \\
\hline & Luffa operculata L. Cogn. & $\mathrm{L}$ & native & NE/no/Cerrado, Atlantic Forest \\
\hline & Momordica charantia L. & $\mathrm{L}$ & naturalized (Asia) & - \\
\hline Dilleniaceae & Curatella americana $\mathrm{L}$. & Ar, S & native & $\begin{array}{c}\text { NE/no/Amazon, Caatinga, } \\
\text { Cerrado, Atlantic Forest }\end{array}$ \\
\hline Dioscoreaceae & Dioscorea trifidaL. f. & $\mathrm{L}$ & native & LC/no/Amazon, Cerrado \\
\hline Equisetaceae & Equisetum giganteum $\mathrm{L}$. & $\mathrm{H}$ & native & NE/no/Cerrado, Atlantic Forest. \\
\hline
\end{tabular}




\begin{tabular}{|c|c|c|c|c|}
\hline \multirow{5}{*}{ Euphorbiaceae } & $\begin{array}{l}\text { Croton antisyphiliticus } \\
\text { Mart. }\end{array}$ & $\begin{array}{c}\mathrm{H}, \mathrm{S} \\
\mathrm{Sb}\end{array}$ & native & $\begin{array}{c}\text { NE/no/Amazon, Caatinga, } \\
\text { Cerrado, Atlantic Forest }\end{array}$ \\
\hline & Croton urucurana Baill. & $\mathrm{Ar}$ & native & $\begin{array}{c}\text { NE/no/Amazon, Cerrado, } \\
\text { Atlantic Forest }\end{array}$ \\
\hline & Euphorbia hirta L. & $\mathrm{h}$ & native & $\begin{array}{c}\text { NE/no/Amazon, Caatinga, } \\
\text { Cerrado, Atlantic Forest, Pampa }\end{array}$ \\
\hline & Manihot esculenta Crantz. & $\mathrm{S}$ & native & NE/no/Amazon, Cerrado \\
\hline & Ricinus communis $\mathrm{L}$. & $S$ & $\begin{array}{l}\text { naturalized } \\
\text { (Africa) }\end{array}$ & 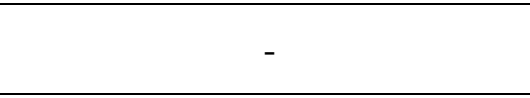 \\
\hline \multirow{16}{*}{ Fabaceae } & $\begin{array}{l}\text { Amburana cearenses } \\
\text { (Allemao) A. C. Sm. }\end{array}$ & $\mathrm{Ar}$ & native & $\begin{array}{l}\text { NT/no/Caatinga, Cerrado, } \\
\text { Atlantic Forest, Pantanal }\end{array}$ \\
\hline & $\begin{array}{c}\text { Anadenanthera colubrina } \\
\text { (Vell.) Brenan }\end{array}$ & $\mathrm{S}, \mathrm{Ar}$ & native & $\begin{array}{c}\text { NE/no/Caatinga, Cerrado, } \\
\text { Atlantic Forest }\end{array}$ \\
\hline & Apuleia ferrea (Mart.) Baill. & $\mathrm{Ar}$ & exotic (Africa) & - \\
\hline & Bauhinia variegata $\mathrm{L}$. & $\mathrm{Ar}$ & $\begin{array}{l}\text { naturalized } \\
\text { (China) }\end{array}$ & - \\
\hline & Cajanus cajan (L.) Huth & $\mathrm{S}$ & Exotic (India) & - \\
\hline & Copaifera langsdorffii Desf. & $\mathrm{Ar}$ & native & $\begin{array}{c}\text { NE/no/Amazon, Caatinga, } \\
\text { Cerrado, Atlantic Forest }\end{array}$ \\
\hline & $\begin{array}{l}\text { Desmodium incanum (Sw.) } \\
\text { DC. }\end{array}$ & $\mathrm{Sb}$ & $\begin{array}{c}\text { naturalized } \\
\text { (America) }\end{array}$ & 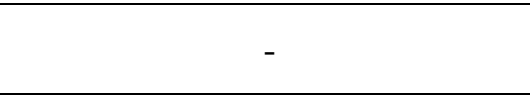 \\
\hline & Dipteryx alata Vogel & $\mathrm{Ar}$ & native & $\begin{array}{c}\text { LC/no /Amazon, Caatinga, } \\
\text { Cerrado }\end{array}$ \\
\hline & Erythrina verna Vell. & $\mathrm{Ar}$ & native & $\begin{array}{c}\text { NE/yes/Amazon, Cerrado, } \\
\text { Atlantic Forest }\end{array}$ \\
\hline & Hymenaea courbaril L. & $\mathrm{Ar}$ & native & $\begin{array}{c}\text { LC/no/Amazon, Caatinga, } \\
\text { Cerrado, Atlantic Forest, Pantanal }\end{array}$ \\
\hline & $\begin{array}{c}\text { Myroxylon balsamum (L.) } \\
\text { Harms }\end{array}$ & $\mathrm{Ar}$ & native & NE/no/Amazon \\
\hline & $\begin{array}{c}\text { Periandra mediterranea } \\
\text { (Vell.) Taub. }\end{array}$ & $\mathrm{S}, \mathrm{Sb}$ & native & $\begin{array}{c}\text { NE/no/Amazon, Caatinga, } \\
\text { Cerrado, Atlantic Forest }\end{array}$ \\
\hline & $\begin{array}{l}\text { Plathymenia reticulata } \\
\text { Benth. }\end{array}$ & $\mathrm{Ar}$ & native & $\begin{array}{c}\text { LC/no/Amazon, Caatinga, } \\
\text { Cerrado, Atlantic Forest }\end{array}$ \\
\hline & $\begin{array}{c}\text { Pterodon emarginatus } \\
\text { Vogel }\end{array}$ & $\mathrm{Ar}$ & native & $\begin{array}{c}\text { NE/no/Amazon, Caatinga, } \\
\text { Cerrado, Pantanal }\end{array}$ \\
\hline & Senna occidentalis (L.) Link & $\mathrm{S}, \mathrm{Sb}$ & native & $\begin{array}{c}\text { NE/no/Amazon, Caatinga, } \\
\text { Cerrado, Atlantic Forest, Pantanal }\end{array}$ \\
\hline & $\begin{array}{c}\text { Stryphnodendron } \\
\text { adstringens (Mart.) Coville }\end{array}$ & $\mathrm{Ar}$ & native & $\begin{array}{c}\text { LC/no/Amazon, Caatinga, } \\
\text { Cerrado }\end{array}$ \\
\hline Gentianaceae & Centaurium erythraea Rafn & $\mathrm{H}$ & $\begin{array}{c}\text { naturalized } \\
\text { (Europe) }\end{array}$ & 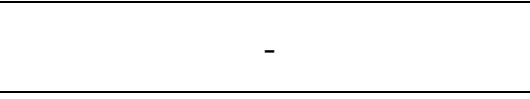 \\
\hline Juncaceae & Juncus effusus L. & $\mathrm{H}$ & native & NE/no/Atlantic Forest \\
\hline \multirow{5}{*}{ Lamiaceae } & $\begin{array}{c}\text { Leonotis nepetifolia (L.) } \\
\text { R.Br. }\end{array}$ & $\mathrm{H}$ & $\begin{array}{c}\text { naturalized } \\
\text { (Africa) }\end{array}$ & 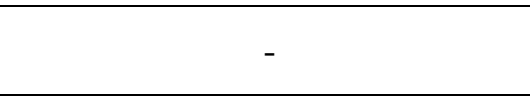 \\
\hline & Mentha canadensis L. & $\mathrm{H}$ & exotic (China) & - \\
\hline & Mentha $x$ officinalis Hull & $\mathrm{S}$ & $\begin{array}{c}\text { exotic } \\
\text { (Mediterranean) }\end{array}$ & - \\
\hline & Mentha pulegium $\mathrm{L}$. & $\mathrm{H}$ & exotic (Europe) & - \\
\hline & Mentha spicata $\mathrm{L}$. & $\mathrm{H}$ & exotic (Europe) & - \\
\hline
\end{tabular}




\begin{tabular}{|c|c|c|c|c|}
\hline & Ocimum basilicum $\mathrm{L}$. & $\mathrm{Sb}$ & $\begin{array}{l}\text { exotic (Asia, } \\
\text { Africa) }\end{array}$ & - \\
\hline & Ocimum gratissimum $\mathrm{L}$. & $\mathrm{Sb}$ & $\begin{array}{l}\text { naturalized (Asia } \\
\text { and Africa) }\end{array}$ & - \\
\hline & Origanum majorana L. & $\mathrm{Sb}$ & exotic (Asia) & - \\
\hline & $\begin{array}{c}\text { Plectranthus barbatus } \\
\text { Andrews }\end{array}$ & $\mathrm{H}$ & exotic (Africa) & - \\
\hline & $\begin{array}{l}\text { Plectranthus amboinicus } \\
\text { (Lour.) Spreng. }\end{array}$ & $\mathrm{H}$ & exotic (Africa) & - \\
\hline & Rosmarinus officinalis L. & $\mathrm{Sb}$ & exotic (Europe) & - \\
\hline & $\begin{array}{l}\text { Tetradenia riparia (Hochst.) } \\
\text { Codd }\end{array}$ & $\mathrm{Ar}$ & exotic (Africa) & - \\
\hline \multirow{2}{*}{ Lauraceae } & Persea americana Mill. & $\mathrm{Ar}$ & $\begin{array}{c}\text { naturalized } \\
\text { (America) }\end{array}$ & - \\
\hline & Cinnamomum verum J.Presl & $\mathrm{Ar}$ & exotic (Asia) & - \\
\hline Loganiaceae & $\begin{array}{c}\text { Strychnos pseudoquina } \\
\text { A.St.-Hil. }\end{array}$ & Ar, S & native & $\begin{array}{c}\text { NE/no/Caatinga, Cerrado, } \\
\text { Atlantic Forest, Pantanal }\end{array}$ \\
\hline \multirow{3}{*}{ Lythraceae } & Lafoensia pacari St. Hil. & $\mathrm{Ar}$ & native & LC/no/Cerrado \\
\hline & Punica granatum L. & $\mathrm{S}, \mathrm{Ar}$ & exotic (Asia) & - \\
\hline & Gossypium herbaceum L. & $\mathrm{S}$ & $\begin{array}{c}\text { naturalized } \\
\text { (America) }\end{array}$ & 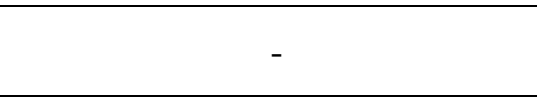 \\
\hline Malpighiaceae & $\begin{array}{c}\text { Byrsonima verbascifolia (L.) } \\
\text { DC. }\end{array}$ & $\mathrm{S}, \mathrm{Ar}$ & native & $\begin{array}{l}\text { NE/no/Amazon, Caatinga, } \\
\text { Cerrado, Atlantic Forest. }\end{array}$ \\
\hline \multirow{2}{*}{ Malvaceae } & Guazuma ulmifolia Lam. & $\mathrm{Ar}$ & native & $\begin{array}{c}\text { NE/no/Amazon, Caatinga, } \\
\text { Cerrado, Atlantic Forest }\end{array}$ \\
\hline & $\begin{array}{c}\text { Helicteres brevispira A.St.- } \\
\text { Hil. }\end{array}$ & $\mathrm{S}$ & native & $\begin{array}{c}\text { NE/no/Amazon, Caatinga, } \\
\text { Cerrado, Atlantic Forest, Pantanal }\end{array}$ \\
\hline Melastomataceae & $\begin{array}{c}\text { Miconia albicans (Sw.) } \\
\text { Triana } \\
\end{array}$ & $\mathrm{S}, \mathrm{Ar}$ & native & $\begin{array}{c}\text { NE/no/Amazon, Caatinga, } \\
\text { Cerrado, Atlantic Forest }\end{array}$ \\
\hline Meliaceae & $\begin{array}{l}\text { Guarea guidonia }(\mathrm{L} .) \\
\text { Sleumer } \\
\end{array}$ & $\mathrm{Ar}$ & native & $\begin{array}{l}\text { NE/no/Amazon, Caatinga, } \\
\text { Cerrado, Atlantic Forest }\end{array}$ \\
\hline \multirow{4}{*}{ Moraceae } & $\begin{array}{c}\text { Brosimum gaudichaudii } \\
\text { Trécul }\end{array}$ & Ar, S & native & $\begin{array}{l}\text { NE/no/Amazon, Caatinga, } \\
\text { Cerrado, Atlantic Forest }\end{array}$ \\
\hline & Dorstenia cayapia Vell. & $\mathrm{H}$ & native & $\begin{array}{c}\text { LC/yes/Caatinga, Cerrado, } \\
\text { Atlantic Forest }\end{array}$ \\
\hline & $\begin{array}{l}\text { Maclura tinctoria (L.) D. } \\
\text { Don ex Steud. }\end{array}$ & $\mathrm{Ar}$ & native & $\begin{array}{c}\text { NE/no/Amazon, Caatinga, } \\
\text { Cerrado, Atlantic Forest, Pantanal }\end{array}$ \\
\hline & Morus alba $\mathrm{L}$. & Ar, S & exotic (China) & - \\
\hline Moringaceae & Moringa oleifera Lam. & $\mathrm{S}$ & exotic (India) & - \\
\hline Musaceae & Musa paradisiaca $\mathrm{L}$. & $\mathrm{H}$ & $\begin{array}{c}\text { naturalized } \\
\text { (Nicaragua) }\end{array}$ & - \\
\hline \multirow{3}{*}{ Myrtaceae } & Eucalyptus citriodora Hook. & $\mathrm{Ar}$ & exotic (Australia) & - \\
\hline & Eucalyptus globulus Labill & $\mathrm{Ar}$ & exotic (Australia) & - \\
\hline & Psidium guajava $\mathrm{L}$. & $\mathrm{Ar}$ & $\begin{array}{c}\text { naturalized } \\
\text { (America) }\end{array}$ & 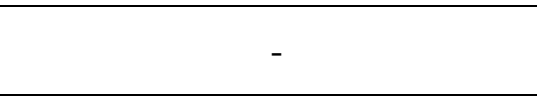 \\
\hline \multirow{2}{*}{ Orchidaceae } & $\begin{array}{c}\text { Cyrtopodium } \\
\text { saintlegerianum Rchb.f. }\end{array}$ & $\mathrm{H}$ & native & $\begin{array}{c}\text { NE/no/Amazon, Caatinga, } \\
\text { Cerrado }\end{array}$ \\
\hline & $\begin{array}{l}\text { Vanilla planifolia Jacks. ex } \\
\text { Andrews }\end{array}$ & $\mathrm{H}$ & native & NE/no/Amazon, Atlantic Forest \\
\hline
\end{tabular}

Boletín Latinoamericano y del Caribe de Plantas Medicinales y Aromáticas / 495 


\begin{tabular}{|c|c|c|c|c|}
\hline Passifloraceae & Passiflora edulis Sims & $\mathrm{L}$ & native & $\begin{array}{c}\text { LC/no/Amazon, Caatinga, } \\
\text { Cerrado, Atlantic Forest, Pantanal }\end{array}$ \\
\hline Phyllanthaceae & Phyllanthus niruri L. & $\mathrm{H}, \mathrm{Sb}$ & native & $\begin{array}{c}\text { NE/no/Amazon, Caatinga, } \\
\text { Cerrado, Atlantic Forest }\end{array}$ \\
\hline Phytolaccaceae & Petiveria alliacea $\mathrm{L}$. & $\mathrm{Sb}$ & $\begin{array}{l}\text { naturalized } \\
\text { (Africa) }\end{array}$ & 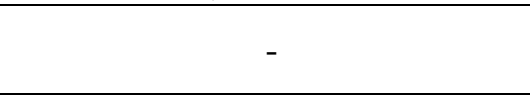 \\
\hline \multirow[t]{2}{*}{ Piperaceae } & Piper aduncum L. & $S$ & native & $\begin{array}{c}\text { NE/no/Amazon, Caatinga, } \\
\text { Cerrado, Atlantic Forest, Pampa, } \\
\text { Pantana }\end{array}$ \\
\hline & Piper nigrum L. & $\mathrm{S}, \mathrm{L}$ & exotic (India) & - \\
\hline Plantaginaceae & Plantago major $\mathrm{L}$. & $\mathrm{H}$ & $\begin{array}{c}\text { naturalized } \\
\text { (Europe) }\end{array}$ & - \\
\hline Proteaceae & $\begin{array}{c}\text { Roupala montana var. } \\
\text { brasiliensis (Klotzsch) K. S. } \\
\text { Edwards }\end{array}$ & $\mathrm{S}, \mathrm{Ar}$ & native & NE/no/Cerrado, Atlantic Forest \\
\hline \multirow{4}{*}{ Poaceae } & Coix lacryma-jobi $\mathrm{L}$. & $\mathrm{S}$ & naturalized (India) & - \\
\hline & $\begin{array}{l}\text { Cymbopogon citratus (DC.) } \\
\text { Stapf }\end{array}$ & $\mathrm{H}$ & naturalized (India) & - \\
\hline & $\begin{array}{l}\text { Cymbopogon nardus (L.) } \\
\text { Rendle }\end{array}$ & $\mathrm{H}$ & $\begin{array}{c}\text { naturalized (Africa } \\
\text { and Asia) }\end{array}$ & - \\
\hline & $\begin{array}{c}\text { Melinis minutiflora } \mathrm{P} . \\
\text { Beauv. }\end{array}$ & $\mathrm{H}$ & $\begin{array}{c}\text { naturalized } \\
\text { (Africa) }\end{array}$ & $\mathrm{C}_{1}$ \\
\hline Polygalaceae & Polygala longicaulis Kunth. & $\mathrm{H}$ & native & $\begin{array}{c}\text { NE/yes/Amazon, Caatinga, } \\
\text { Cerrado, Atlantic Forest, Pampa, } \\
\text { Pantanal }\end{array}$ \\
\hline Polygonaceae & Rumex acetosa $\mathrm{L}$ & $\mathrm{H}$ & naturalized (Asia) & - \\
\hline Pteridaceae & $\begin{array}{c}\text { Adiantum capillus-veneris } \\
\text { L. }\end{array}$ & $\mathrm{H}$ & native & NE/no/Atlantic Forest \\
\hline \multirow{2}{*}{ Rosaceae } & Prunus domestica L. & $\mathrm{Ar}$ & exotic (China) & - \\
\hline & Rosa alba L. & $\mathrm{S}$ & exotic (Asia) & - \\
\hline \multirow{6}{*}{ Rubiaceae } & $\begin{array}{l}\text { Alibertia edulis (Rich.) } \\
\text { A.Rich. }\end{array}$ & $\mathrm{S}, \mathrm{Ar}$ & native & NE/no/Amazon, Cerrado \\
\hline & Genipa americana $\mathrm{L}$. & $\mathrm{S}, \mathrm{Ar}$ & native & $\begin{array}{c}\text { LC/no/Amazon, Caatinga, } \\
\text { Cerrado, Atlantic Forest, Pantanal }\end{array}$ \\
\hline & Morinda citrifolia $\mathrm{L}$. & $\mathrm{S}$ & exotic (Asia) & - \\
\hline & $\begin{array}{l}\text { Palicourea coriacea } \\
\text { (Cham.) K.Schum }\end{array}$ & $\mathrm{S}, \mathrm{Sb}$ & native & $\begin{array}{c}\text { NE/no/Amazon, Caatinga, } \\
\text { Cerrado }\end{array}$ \\
\hline & Palicourea rigida Kunth. & $\mathrm{S}, \mathrm{Sb}$ & native & $\begin{array}{c}\text { NE/no/Amazon, Caatinga, } \\
\text { Cerrado, Atlantic Forest, Pampa }\end{array}$ \\
\hline & $\begin{array}{l}\text { Rudgea viburnoides (Cham.) } \\
\text { Benth. }\end{array}$ & $\mathrm{S}$ & native & $\begin{array}{c}\text { NE/no/Amazon, Caatinga, } \\
\text { Cerrado }\end{array}$ \\
\hline \multirow{5}{*}{ Rutaceae } & Citrus aurantium L. & $\mathrm{Ar}$ & exotic (Asia) & - \\
\hline & $\begin{array}{l}\text { Citrus aurantiifolian } \\
\text { (Christm.) Swingle }\end{array}$ & $\mathrm{Ar}$ & exotic (Asia) & - \\
\hline & Citrus limon (L.) Osbeck & $\mathrm{Ar}$ & naturalized (Asia) & - \\
\hline & Citrus sinensis (L.) Osbeck & $\mathrm{Ar}$ & exotic (Asia) & - \\
\hline & Ruta graveolens L. & $\mathrm{H}$ & Exotic (Europe) & - \\
\hline
\end{tabular}




\begin{tabular}{|c|c|c|c|c|}
\hline Salicaceae & Casearia sylvestris $\mathrm{Sw}$. & $\begin{array}{l}\mathrm{Ar}, \mathrm{S} \\
\mathrm{Sb}\end{array}$ & native & $\begin{array}{c}\text { NE/no/Amazon, Caatinga, } \\
\text { Cerrado, Atlantic Forest, Pampa, } \\
\text { Pantanal }\end{array}$ \\
\hline Sapindaceae & $\begin{array}{l}\text { Dilodendron bipinnatum } \\
\text { Radlk. }\end{array}$ & $\mathrm{Ar}$ & native & $\begin{array}{c}\text { LC/no/Amazon, Cerrado, } \\
\text { Atlantic Forest }\end{array}$ \\
\hline Sapotaceae & $\begin{array}{l}\text { Pouteria caimito (Ruiz Pav.) } \\
\text { Radlk. }\end{array}$ & $\mathrm{Ar}$ & native & $\begin{array}{c}\text { NE/no/Amazon, Cerrado, } \\
\text { Atlantic Forest }\end{array}$ \\
\hline Siparunaceae & Siparuna guianensis Aublet & $\mathrm{S}, \mathrm{Ar}$ & native & $\begin{array}{c}\text { NE/no/Amazon, Caatinga, } \\
\text { Cerrado, Atlantic Forest, Pantanal }\end{array}$ \\
\hline Smilacaceae & Smilax brasiliensis Spreng. & $\begin{array}{l}\mathrm{S}, \mathrm{Sb} \\
\mathrm{L}\end{array}$ & $\begin{array}{c}\text { native and } \\
\text { endemic to Brazil }\end{array}$ & NE/yes/Cerrado \\
\hline \multirow{4}{*}{ Solanaceae } & Atropa belladonna L. & $\mathrm{S}$ & exotic (Australia) & - \\
\hline & Solanum cernuum Vell. & $\mathrm{S}$ & $\begin{array}{c}\text { native and } \\
\text { endemic to Brazil }\end{array}$ & NE/yes/Cerrado, Atlantic Forest \\
\hline & Solanum lycocarpum St. Hil. & Ar, S & native & NE/no/Cerrado, Atlantic Forest \\
\hline & Solanum paniculatum $\mathrm{L}$. & $\mathrm{S}$ & native & $\begin{array}{c}\text { NE/no/Amazon, Caatinga, } \\
\text { Cerrado, Atlantic Forest }\end{array}$ \\
\hline Urticaceae & $\begin{array}{c}\text { Cecropia pachystachya } \\
\text { Trécul }\end{array}$ & $\mathrm{H}$ & native & $\begin{array}{c}\text { NE/no/Amazon, Caatinga, } \\
\text { Cerrado, Atlantic Forest, Pantanal }\end{array}$ \\
\hline Verbenaceae & Lantana trifolia $\mathrm{L}$. & $\mathrm{S}$ & native & $\begin{array}{c}\text { NE/no/Amazon, Cerrado, } \\
\text { Atlantic Forest }\end{array}$ \\
\hline \multirow{3}{*}{ Vochysiaceae } & Vochysia elliptica Mart. & $\mathrm{Ar}$ & native & $\begin{array}{c}\text { NE/yes/Amazon, Caatinga, } \\
\text { Cerrado, Atlantic Forest, Pantanal }\end{array}$ \\
\hline & Qualea grandiflora Mart. & $\mathrm{S}, \mathrm{Ar}$ & native & $\begin{array}{c}\text { NE/no/Amazon, Caatinga, } \\
\text { Cerrado, Atlantic Forest }\end{array}$ \\
\hline & Qualea parviflora Mart. & $\mathrm{S}, \mathrm{Ar}$ & native & $\begin{array}{c}\text { NE/no/Amazon, Caatinga, } \\
\text { Cerrado, Atlantic Forest }\end{array}$ \\
\hline \multirow{3}{*}{ Zingiberaceae } & Curcuma longa $\mathrm{L}$. & $\mathrm{H}$ & exotic (India) & - \\
\hline & $\begin{array}{l}\text { Curcuma zedoaria } \\
\text { (Christm.) Roscoe }\end{array}$ & $\mathrm{H}$ & exotic (Asia) & - \\
\hline & Zingiber officinale Roscoe & $\mathrm{H}$ & exotic (Asia) & - \\
\hline
\end{tabular}

The richest genera in species were Allium L., Citrus L. and Mentha L., all with four species each. In other ethnobotanical studies, in a Cerrado area, similar results were found, such as those of Amorozo (2002); Borba \& Macedo (2006); Silva \& Proença (2007); Alves \& Povh (2013); Ferreira et al. (2015) and Guimarães et al. (2019).

Among the medicinal species mentioned, most are native $(55,11 \%, \mathrm{n}=97)$, followed by exotics $(28,40 \%, \mathrm{n}=50)$ and naturalized $(16.47 \%, \mathrm{n}=29)$ (Table No. 1). Among the native ones eight are endemic to Brazil: Adenocalymma nodosum, Cayaponia tayuya, Dorstenia cayapia, Jacaranda rufa, Smilax brasiliensis, Solanum cernuum,
Vochysia elliptica and Zeyheria montana. The use of medicinal species, the majority of which are native, has also been recorded by other ethnobotanical research (Oliveira et al., 2010; Cunha \& Bortolotto, 2011; Leandro et al., 2017; Guimarães et al., 2019). There was a predominance of herbaceous habit (72), followed by arboreal (59), shrub (55), sub-shrub (23) and liana (14). A similar result was found in the ethnobotanical study with medicinal plants carried out in rural settlements in southwest Goiás (Silva et al., 2010).

Most of the 93 native species $(91.76 \%)$ are found in the Cerrado phytogeographic domain. However, these species are not exclusive to this 
domain and they are shared mainly with the Atlantic Forest (74.12\%), Amazon (67.06\%) and Caatinga (65.88\%) (Figure No. 3). Among these species, four are exclusive to a Brazilian phytogeographic domain, being Aristolochia esperanzae, Gomphrena arborescens and Lafoensia pacari for the Cerrado; Sambucus australis and Myrocarpus frondosus for the Atlantic Forest, and Myroxylon balsamum for the Amazon.

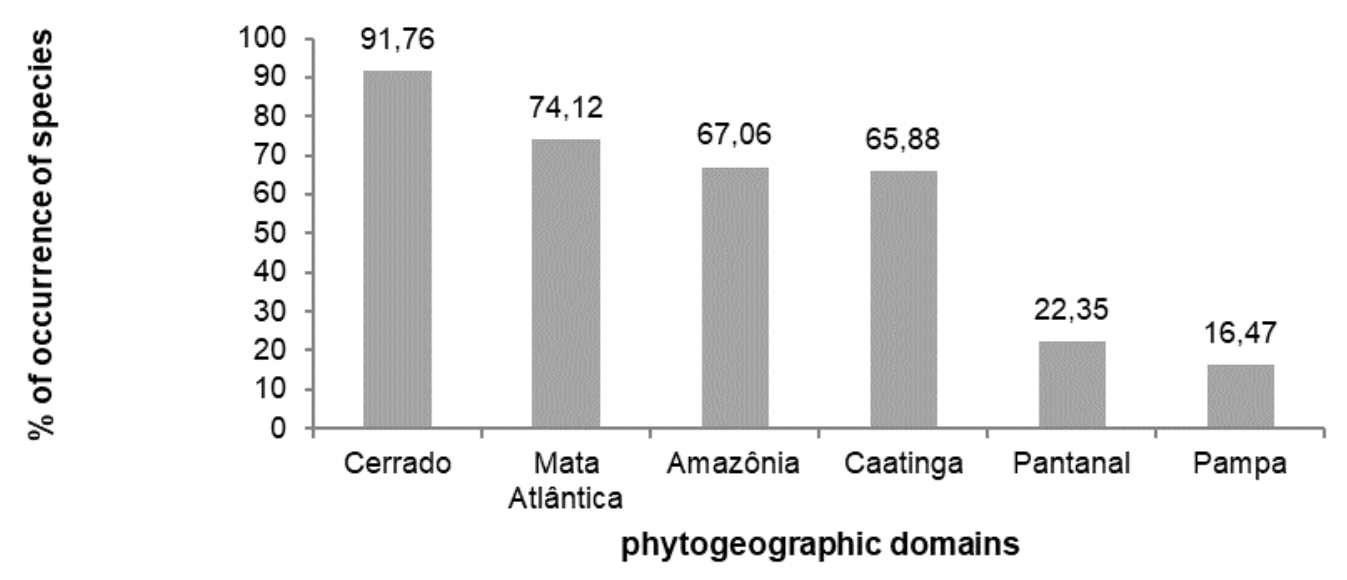

Figure No .3

Percentage of medicinal plant species used in the Boa Esperança Settlement in Piracanjuba, GO, according to the occurrence in the Brazilian phytogeographic domain

Regarding threat status (Flora do Brasil, 2020), most of the native medicinal species mentioned were not evaluated $(40 \%, n=39)$ (Table No. 1). Therefore, it is important to expand the studies to evaluate the threat status of these species to establish future conservation strategies. For Baccharis crispa, Cecropia pachystachya and Vochysia elliptica this evaluation is ongoing. For Xylopia aromatica, Zeyheria montana, Maytenus ilicifolia, Mikania glomerata, Myracrodruon urundeuva, Dioscorea trifida, Dipteryx alata, Stryphnodendron adstringens, Hymenaea courbaril, Genipa americana, Dilodendron bipinnatum and Terminalia argentea status is least concern (LC), while for Lychnophora ericoides, Amburana cearenses and Handroanthus impetiginosus the status is near threated (NT), requiring studies to prevent possible risks of extinction. The species Anemopaegma arvense is in the threat category as endangered $(\mathrm{EN})$, being at a very high risk of extinction of nature. This species was found at the study site in the reproductive period, the which demonstrates the importance of ethnobotanical studies for the conservation of plant species.
There was a predominance of use of plants to treat diseases related to airway problems $(77 \%)$ and digestive system $(75 \%)$, followed by diseases associated with inflammation and pain $(50 \%)$ and dermatological problems (33\%) (Table No. 2; Figure No. 4). Diseases related to the gastrointestinal and respiratory system are among the most cited in other ethnobotanical studies in the country (Pinto et al., 2006; Silva et al., 2010; Guimarães et al., 2019). In the Northeast region of Brazil, the most treated disorders by medicinal plants are gastrointestinal and respiratory tract disorders (Rodrigues \& Andrade, 2014; Brito et al., 2017). The lack of basic sanitation may also justify the large number of citations for the categories of disorders of the digestive and respiratory systems.

As for the part of the plant most used for the preparation of home remedies, the leaves $(54.49 \%)$ were mentioned in decreasing order, followed by the root $(17.98 \%)$ and barks, stem and fruit barks (17.42\%). Other parts were mentioned, such as seeds, shoots, resins and the entire plant. In addition, it was mentioned the combination with other medicinal species and the use of other ingredients in the 
preparation, such as: vinegar, alcohol, honey and wines (Table No. 2). The greater use of leaves for the preparation of herbal medicines can be justified because it contains most of the active ingredients of the plants, in addition to its greater availability throughout the year (Vasquez et al., 2014; Costa \& Marinho, 2016; Gomes \& Lima, 2017).

Table No. 2

Recommendation of the use of plants, used part and instructions for use and/or preparation of medicinal plants used in the Boa Esperança rural settlement of Piracanjuba, GO

\begin{tabular}{|c|c|c|c|}
\hline Species & Recommendation & Used part & $\begin{array}{c}\text { Instructions for use and/or } \\
\text { preparation }\end{array}$ \\
\hline Achillea millefolium & Fungicide & leaves & Infusion \\
\hline Achyrocline satureioides & $\begin{array}{l}\text { Dysentery and digestive } \\
\text { problems }\end{array}$ & leaves, branches & Infusion \\
\hline Acmella oleraceae & $\begin{array}{l}\text { Antifungal, antiseptic, } \\
\text { antiviral, diuretic and } \\
\text { immune system } \\
\text { stimulant }\end{array}$ & leaves & Food and tea \\
\hline Adenocalymma nodosum & $\begin{array}{l}\text { Laxative, purgative, } \\
\text { blood purifier }\end{array}$ & leaves & Infusion \\
\hline Adiantum capillus-veneris & Flu, cold, cough & leaves & Tea \\
\hline Ageratum conyzoides & $\begin{array}{l}\text { Analgesic, prickly heat, } \\
\text { indigestion, menstrual } \\
\text { cramps }\end{array}$ & leaves and roots & Infusion, baths \\
\hline Alibertia edulis & $\begin{array}{l}\text { Anti-inflammatory } \\
\text { urinary tract, diarrhea, } \\
\text { hemorrhoids }\end{array}$ & leaves & Tea por infusion and syrup \\
\hline Allium cepa & Flu, cough, throat & bulb & $\begin{array}{l}\text { Syrup with lemon, saffron, garlic, } \\
\text { chives, honey and water }\end{array}$ \\
\hline Allium fistulosum & Flu, cough, throat & leaves & $\begin{array}{l}\text { Syrup with lemon, saffron, garlic, } \\
\text { chives, honey and water }\end{array}$ \\
\hline Allium porrum & Infections & bulb & Spice \\
\hline Allium sativum & $\begin{array}{l}\text { Flu, cough, throat, anti- } \\
\text { inflammatory }\end{array}$ & bulb & $\begin{array}{l}\text { Syrup with lemon, saffron, garlic, } \\
\text { chives, honey and water }\end{array}$ \\
\hline Aloe vera & $\begin{array}{l}\text { Healing, emollient, } \\
\text { antimicrobial, } \\
\text { hemorrhoids, gastritis, } \\
\text { ulcer }\end{array}$ & leaves & Tea \\
\hline Alternanthera brasiliana & $\begin{array}{l}\text { Anti-inflammatory, } \\
\text { healinge, painkiller }\end{array}$ & leaves & Tea, plaster \\
\hline Amburana cearenses & Circulatory problems & bast, seeds & Infusion \\
\hline Anadenanthera colubrina & Bronchitis, asthma & bast, resin & Tea, syrup \\
\hline Anemopagma arvense & Aphrodisiac, kidneys & $\begin{array}{l}\text { leaves, root, } \\
\text { whole plant }\end{array}$ & Infusion, pill \\
\hline Annona muricata & Antitumor & leaves, fruits & Tea and juice \\
\hline Apuleia ferrea & $\begin{array}{l}\text { Diabete, arthritis, } \\
\text { rheumatism }\end{array}$ & bard, seeds & Decoction, pill \\
\hline
\end{tabular}

Boletín Latinoamericano y del Caribe de Plantas Medicinales y Aromáticas / 499 


\begin{tabular}{|c|c|c|c|}
\hline Aristolochia esperanzae & $\begin{array}{l}\text { Stomach burn, } \\
\text { heartburn, indigestion }\end{array}$ & leaves & Infusion \\
\hline Aristolochia trilobata & $\begin{array}{l}\text { Diarrhea, dysentery, } \\
\text { ulcer }\end{array}$ & whole plant & Infusion \\
\hline Artemisia absinthium & $\begin{array}{l}\text { Herpes, erysipelas, skin } \\
\text { wounds and diabetes, } \\
\text { stomach disorders }\end{array}$ & leaves, root & Infusion, maceration \\
\hline Atropa belladonna & erysipelas & flowers & Bath \\
\hline Baccharis crispa & $\begin{array}{l}\text { Liver problems, stomach } \\
\text { disorders, intestinais, } \\
\text { anti-inflammatory, } \\
\text { diuretic, digestive, } \\
\text { antianemic, } \\
\text { antiasthmatic, antibiotic, } \\
\text { antidiarrheal, } \\
\text { antidispeptic, antigripal, } \\
\text { anti-hydropic, anti- } \\
\text { rheumatic, laxative, } \\
\text { sweating and } \\
\text { deworming, diabetes }\end{array}$ & leaves & Infusion, powder, pill \\
\hline Bauhinia variegata & $\begin{array}{l}\text { Diabetes, chest pain, } \\
\text { heart }\end{array}$ & flower & Infusion \\
\hline Beta vulgaris & Expectorant & tuber & $\begin{array}{l}\text { Put beet and onion pieces, let it rest, } \\
\text { and drink the syrup }\end{array}$ \\
\hline Bidens pilosa & $\begin{array}{l}\text { Hepatite, anemia, } \\
\text { icterus, healing, wounds, } \\
\text { mycoses }\end{array}$ & $\begin{array}{l}\text { leaves, whole } \\
\text { plant }\end{array}$ & Infusion, baths \\
\hline Bixa orellana & $\begin{array}{l}\text { Anemia, anti- } \\
\text { inflammatory }\end{array}$ & leaves, fruits & Infusion, powder \\
\hline Brassica sylvestris & Gastritis & leaves & Juice \\
\hline Brosimum gaudichaudii & $\begin{array}{l}\text { Vitiligo, ulcers, } \\
\text { dewormer }\end{array}$ & $\begin{array}{l}\text { bark of roots, } \\
\text { fruits, leaves }\end{array}$ & $\begin{array}{l}\text { Decoction of roots and leaves and use } \\
\text { in the bath }\end{array}$ \\
\hline Byrsonima verbascifolia & Cold, antioxidant & fruits & Food \\
\hline Cajanus cajan & $\begin{array}{l}\text { Anti-inflammatory, } \\
\text { sinusitis }\end{array}$ & seed & Food \\
\hline Carica papaya & $\begin{array}{l}\text { Asthma, flu, diabetes, } \\
\text { dewormer and to } \\
\text { eliminate warts }\end{array}$ & $\begin{array}{l}\text { latex, seeds, } \\
\text { flowers }\end{array}$ & $\begin{array}{l}\text { Fresh latex is used to eliminate warts. } \\
\text { It is common to use dry and ground } \\
\text { seeds in the form of tea as a dewormer } \\
\text { and for influenza }\end{array}$ \\
\hline Casearia sylvestris & $\begin{array}{l}\text { In the treatment of } \\
\text { pulmonary emphysema, } \\
\text { gastritis and Ulcer, } \\
\text { spine, rheumatism, } \\
\text { diarrhea, hemorrhoids }\end{array}$ & leaves & Maceration \\
\hline Catharanthus roseus & Fungicide, cancer & flowers & Infusion \\
\hline Cayaponia tayuya & Against pain in general & roots & Decoction \\
\hline
\end{tabular}




\begin{tabular}{|c|c|c|c|}
\hline & and as a blood purifier & & \\
\hline Cecropia pachystachya & $\begin{array}{l}\text { Diabetes, Kidneys, pain } \\
\text { in general, cancer }\end{array}$ & $\begin{array}{l}\text { broto, leaves, } \\
\text { bast }\end{array}$ & Tea, cream \\
\hline Celtis iguanea & Kidney problems & leaves & Infusion \\
\hline Centaurium erythraea & $\begin{array}{l}\text { Liver, indigestion, } \\
\text { gallbladder, } \\
\text { gastrointestinal disorder }\end{array}$ & leaves & Infusion \\
\hline Cinnamomum verum & Cold, sore throat & $\begin{array}{l}\text { leaves, bark e } \\
\text { bast }\end{array}$ & $\begin{array}{l}\text { Tea, hot chocolate with other } \\
\text { ingredients (egg, alfavaca, honey) }\end{array}$ \\
\hline Citrus aurantiifolian & $\begin{array}{l}\text { In the treatment of } \\
\text { sinusitis and to regulate } \\
\text { blood pressure }\end{array}$ & fruits & $\begin{array}{l}\text { Remove the bark of the fruit; after } \\
\text { washing, place it in a glass jar with } \\
\text { alcohol and smell }\end{array}$ \\
\hline Citrus aurantium & Constipation & fruits & Food \\
\hline Citrus limon & $\begin{array}{l}\text { Gastritis, antiseptic use } \\
\text { indicated mainly to } \\
\text { prevent infectious } \\
\text { diseases, flu, cold, } \\
\text { expectorant }\end{array}$ & fruit juice & $\begin{array}{l}\text { Drink the fruit broth with water. Drink } \\
\text { with honey, saffron, garlic and lemon. }\end{array}$ \\
\hline Citrus sinensis & indigestion & bark & $\begin{array}{l}\text { Peel with water, coffee powder, boil } \\
\text { and drink }\end{array}$ \\
\hline Cochlospermum regium & Infections, blood purifier & root & Tea \\
\hline Coix lacryma-jobi & $\begin{array}{l}\text { Diuretic, antiseptic } \\
\text { respiratory and urinary } \\
\text { tract and antirheumatic }\end{array}$ & leaves, fruits & Infusion \\
\hline Copaifera langsdorffii & Pneumonia, healing & oil & $\begin{array}{l}\text { Burn the oil in alcohol and apply to } \\
\text { the pain site }\end{array}$ \\
\hline Costus spiralis & $\begin{array}{l}\text { Reins, kidney stones, } \\
\text { tumors }\end{array}$ & stem, leaves & Infusion \\
\hline Croton antisyphiliticus & $\begin{array}{l}\text { Infections, infection in } \\
\text { the uterus, ovary, cyst, } \\
\text { fibroid, discharge, } \\
\text { gastritis, ulcer, } \\
\text { hemorrhoid, diarrhea, } \\
\text { scarring and as a blood } \\
\text { purifier }\end{array}$ & $\begin{array}{l}\text { root, leaves, } \\
\text { whole plant }\end{array}$ & Infusion, decoction \\
\hline Croton urucurana & $\begin{array}{l}\text { Uterine infection, } \\
\text { healing, infections, } \\
\text { gastritis, to eliminate } \\
\text { erysipelass and skin } \\
\text { rashes }\end{array}$ & bark, sap, resin & Tea \\
\hline Cucumis anguria & aphrodisiac & fruits, leaves & Food \\
\hline Cucurbita pepo & Worms & seeds & Toasting and grinding \\
\hline Cuminum cyminum & Increases immunity & Leaves, fruits & Tea \\
\hline Curatella americana & Kidney infections & leaves & Infusion \\
\hline Curcuma longa & $\begin{array}{l}\text { In the treatment of cough } \\
\text { and diseases of the }\end{array}$ & rhizomes & Tea or syrup \\
\hline
\end{tabular}




\begin{tabular}{|c|c|c|c|}
\hline & $\begin{array}{l}\text { respiratory tract, } \\
\text { antifungal, bactericidal, } \\
\text { anti-caries, depression, } \\
\text { cancer }\end{array}$ & & \\
\hline Curcuma zedoaria & $\begin{array}{l}\text { Vermifuge, vomit, } \\
\text { digestive }\end{array}$ & leaves, root & Tea, plaster \\
\hline Cymbopogon citratus & $\begin{array}{l}\text { Soothing, antidepressant } \\
\text { in the treatment of flu }\end{array}$ & leaves & Infusion \\
\hline Cymbopogon nardus & Repellent & leaves & Infusion by alcohol \\
\hline $\begin{array}{l}\text { Cyrtopodium } \\
\text { saintlegerianum }\end{array}$ & $\begin{array}{l}\text { Stomach and boil } \\
\text { problems }\end{array}$ & sap, bulb, root & Syrup \\
\hline Dasyphyllum brasiliense & Kidney problems & leaves & Infusion \\
\hline Daucus carota & Increases immunity & root & $\begin{array}{l}\text { Juice beaten with passion fruit, lemon, } \\
\text { cabbage and orange or in food }\end{array}$ \\
\hline Desmodium incanum & $\begin{array}{l}\text { Kidney stones, kidney } \\
\text { problems }\end{array}$ & leaves & Infusion \\
\hline Dilodendron bipinnatum & $\begin{array}{l}\text { Body pain, low back } \\
\text { pain treatment, back } \\
\text { problems }\end{array}$ & leaves, bark & Tea por decoction and infusion \\
\hline Dioscorea trifida & $\begin{array}{l}\text { Blood purifier, female } \\
\text { fertility }\end{array}$ & rhizome & Food \\
\hline Dipteryx alata & $\begin{array}{l}\text { Kidney problems, } \\
\text { kidney stones }\end{array}$ & bast fruits, seeds & Infusion \\
\hline Dorstenia cayapia & $\begin{array}{l}\text { Anti-inflammatory, } \\
\text { diarrhea }\end{array}$ & root & Food \\
\hline Dysphania ambrosioides & $\begin{array}{l}\text { Anti-inflammatory, } \\
\text { healing, anesthetizing, } \\
\text { worms }\end{array}$ & leaves, fruits & $\begin{array}{l}\text { Maceration tea; plaster of the leaf with } \\
\text { little vinegar (put on the wound); beat } \\
\text { the leaf in the blender with mint and } \\
\text { milk; shaped candy }\end{array}$ \\
\hline Echinodorus grandiflorus & $\begin{array}{l}\text { Kidney stones, anti- } \\
\text { inflammatory, liver } \\
\text { problems }\end{array}$ & leaves & Infusion \\
\hline Equisetum giganteum & Kidney problems & whole plant & Tea \\
\hline Eruca sativa & Infections & root & Tea \\
\hline Eryngium foetidum & Digestive, infections & whole plant & Spice \\
\hline Erythrina verna & Hepatite, soothing & bast, bark & Infusion \\
\hline Eucalyptus citriodora & Repellent, flu, sinusitis & leaves & Infusion \\
\hline Eucalyptus globulus & Pneumonia & leaves & Infusion \\
\hline Euphorbia hirta & Hemorrhage, warts & root, latex & Tea \\
\hline Foeniculum vulgare & $\begin{array}{l}\text { Flu and blood pressure } \\
\text { regulator }\end{array}$ & $\begin{array}{l}\text { seeds, leaves and } \\
\text { root }\end{array}$ & Infusion tea \\
\hline Genipa americana & $\begin{array}{l}\text { Kidney infections, } \\
\text { expectorant }\end{array}$ & bast & Infusion, syrup \\
\hline Gomphrena arborescens & Fever and diarrhea & root & Decoction \\
\hline Gossypium herbaceum & $\begin{array}{l}\text { Antibiotic, infections } \\
\text { treatment, kidney }\end{array}$ & leaves & Infusion, maceration \\
\hline
\end{tabular}

Boletín Latinoamericano y del Caribe de Plantas Medicinales y Aromáticas / 502 


\begin{tabular}{|c|c|c|c|}
\hline & infection, cough & & \\
\hline Guarea guidonia & Stomach problems & leaves e fruits & Decoction \\
\hline Guazuma ulmifolia & $\begin{array}{l}\text { Spine problems, hair } \\
\text { tonic, astringent and } \\
\text { sweat }\end{array}$ & sap & Shampoo, cream \\
\hline Hancornia speciosa & Ulcer & resin, fruits & Food \\
\hline $\begin{array}{l}\text { Handroanthus } \\
\text { impetiginosus }\end{array}$ & $\begin{array}{l}\text { Anti-inflammatory and } \\
\text { antibacterial, bronchitis, } \\
\text { asthma }\end{array}$ & bast & Infusion \\
\hline Helicteres brevispira & Blood purifier & leaves, roots & Tea, infusion \\
\hline Himatanthus obovatus & Blood purifier & rhizome & Food \\
\hline Hymenaea courbaril & $\begin{array}{l}\text { Bronchitis, throat } \\
\text { infection, bones, prostate }\end{array}$ & bark, bast, resin & Maceration \\
\hline Impatiens balsamina & Skin lesions & leaves, stems & $\begin{array}{l}\text { Punched leaves and stems can be } \\
\text { applied directly to the affected parts }\end{array}$ \\
\hline Jacaranda rufa & $\begin{array}{l}\text { Masks, wounds, healing, } \\
\text { blood purifier }\end{array}$ & $\begin{array}{l}\text { leaves, root, } \\
\text { whole plant }\end{array}$ & Bath, Tea \\
\hline Juncus effusus & Female infections & root & Infusion by alcohol \\
\hline Justicia pectoralis & Vertigo & leaves & Infusion, decoction \\
\hline Kalanchoe laetivirens & Gastritis, ulcer & leaves & Maceration \\
\hline Kalanchoe pinnata & $\begin{array}{l}\text { Natural expectorant, } \\
\text { gastritis, ulcer }\end{array}$ & leaves & Maceration, juice \\
\hline Lafoensia pacari & $\begin{array}{l}\text { Diarrhea, intestinal } \\
\text { problems }\end{array}$ & bark, bast & Infusion \\
\hline Lantana trifolia & $\begin{array}{l}\text { Belly pain with } \\
\text { infection, cough }\end{array}$ & whole plant & Tea \\
\hline Leonotis nepetifolia & $\begin{array}{l}\text { Diarrhea, asthma, } \\
\text { rheumatism }\end{array}$ & whole plant, root & Infusion \\
\hline Luffa operculata & Sinusitis & $\begin{array}{l}\text { fibrous fruit } \\
\text { loofah }\end{array}$ & $\begin{array}{l}\text { Put a coffee spoon of salt in a teacup } \\
\text { of water. Peel the buchinha and cut a } \\
\text { piece with } 2 \mathrm{~cm} \text { and put in the cup of } \\
\text { water and salt, letting it rest for } 5 \text { days } \\
\text { and strain. Drip } 2 \text { drops into each } \\
\text { nostril, morning and evening, without } \\
\text { blowing your nose, letting it flow } \\
\text { naturally }\end{array}$ \\
\hline Lychnophora ericoides & $\begin{array}{l}\text { Bruises, relieve swelling } \\
\text { and pain in the legs, } \\
\text { repellent and anti- } \\
\text { inflammatory }\end{array}$ & whole plant & Infusion in alcohol, soap, cream, gel \\
\hline Maclura tinctoria & Infections in the tooth & latex & Skin \\
\hline Mandevilla velame & $\begin{array}{l}\text { Anti-inflammatory, } \\
\text { infections and blood } \\
\text { purifying }\end{array}$ & root & Decoction \\
\hline
\end{tabular}




\begin{tabular}{|c|c|c|c|}
\hline Mangifera indica & Bronchitis & leaves & $\begin{array}{l}\text { The leaves must be dried in the sun, in } \\
\text { a ventilated place and without } \\
\text { humidity, and then they must be } \\
\text { stored in cloth or paper bags. Making } \\
\text { tea by infusion }\end{array}$ \\
\hline Manihot esculenta & Strengthen bones & leaves & Flour, food \\
\hline Matricaria recutita & $\begin{array}{l}\text { Soothing, indigestion } \\
\text { and intestinal problems }\end{array}$ & flowers & Infusion \\
\hline Mauritia flexиosa & Snake bite & leaves & $\begin{array}{l}\text { Apply the juice of the leaves to the } \\
\text { bite }\end{array}$ \\
\hline Maytenus ilicifolia & Reins, kidney stones & leaves & Infusion \\
\hline Melinis minutiflora & Rheumatism & leaves & Infusion \\
\hline Mentha canadensis & Flu and asthma & leaves & Infusion \\
\hline Mentha pulegium & Cure navel and jaundice & leaves & Infusion \\
\hline Mentha spicata & Soothing and gases & leaves & Infusion \\
\hline Mentha $x$ officinalis & $\begin{array}{l}\text { Labyrinthitis, high blood } \\
\text { pressure }\end{array}$ & leaves & Infusion \\
\hline Miconia albicans & $\begin{array}{l}\text { Anti-inflammatory, back } \\
\text { pain, joints, for arthritis, } \\
\text { osteoarthritis, urine } \\
\text { infection }\end{array}$ & leaves, roots & Maceration, cream \\
\hline Mikania glomerata & $\begin{array}{l}\text { Pneumonia, cough, } \\
\text { Bronchitis and } \\
\text { hoarseness, throat } \\
\text { infection }\end{array}$ & leaves & Infusion, syrup \\
\hline Momordica charantia & $\begin{array}{l}\text { Diabetes, roundworm, } \\
\text { fungicide, bactericide, } \\
\text { repellent }\end{array}$ & leaves & $\begin{array}{l}\text { Use fresh leaf juice diluted with water. } \\
\text { Put it in alcohol, boil it in water and } \\
\text { let it repel insects. Shampoo, soap }\end{array}$ \\
\hline Morinda citrifolia & $\begin{array}{l}\text { In the treatment of } \\
\text { bedsores and various } \\
\text { diseases }\end{array}$ & fruits, leaves & Bath, juice, maceration \\
\hline Moringa oleifera & Anemia, malnutrition & leaves, flowers & Infusion \\
\hline Morus alba & $\begin{array}{l}\text { Treatment of thrush, } \\
\text { menopause, natural } \\
\text { hormone, kidney } \\
\text { problems }\end{array}$ & leaves, fruit & Tea, juice \\
\hline Musa paradisiaca & $\begin{array}{l}\text { Clear lung, bronchitis, } \\
\text { pneumonia and hepatitis }\end{array}$ & bud & Syrup \\
\hline Myracrodruon urundeuva & $\begin{array}{l}\text { Antimicrobial, } \\
\text { erysipelas, blood } \\
\text { purifier, mycoses, some } \\
\text { types of cancer, } \\
\text { cleansing the skin, } \\
\text { itches, acne, spots on the } \\
\text { skin, diarrhea, } \\
\text { disinfection of wounds } \\
\text { and fractures }\end{array}$ & bast & $\begin{array}{l}\text { Bottled, external application in the } \\
\text { form of antiseptic, for fractures and } \\
\text { exposed wounds. In the form of } \\
\text { lotions, gels or soaps. }\end{array}$ \\
\hline
\end{tabular}




\begin{tabular}{|c|c|c|c|}
\hline Myroxylon balsamum & Infections & leaves, bast & Tea \\
\hline Ocimum basilicum & Soothing & leaves & Infusion \\
\hline Ocimum gratissimum & $\begin{array}{l}\text { Flu, sinusitis, } \\
\text { expectorant, lung } \\
\text { problems }\end{array}$ & leaves & $\begin{array}{l}\text { Infuse in the preparation of anti-flu } \\
\text { baths. Beat the yolk with cinnamon, } \\
\text { honey and add the alfavaca tea }\end{array}$ \\
\hline Operculina macrocarpa & Blood purifying & rhizome & Food \\
\hline Origanum majorana & Indigestion, constipation & leaves & Infusion, spice \\
\hline Palicourea coriacea & $\begin{array}{l}\text { Treats skin diseases, } \\
\text { lowers blood pressure } \\
\text { and helps treat syphilis } \\
\end{array}$ & leaves, bark & Decoction \\
\hline Palicourea rigida & $\begin{array}{l}\text { Heart problems, } \\
\text { circulation, wounds, } \\
\text { prevention of stretch } \\
\text { marks }\end{array}$ & leaves & Infusion \\
\hline Passiflora edulis & Immunity, soothing & fruits & Juice \\
\hline Peltastes peltatus & $\begin{array}{l}\text { Uterus infection, female } \\
\text { infertility }\end{array}$ & leaves, stalk & Infusion \\
\hline Periandra mediterranea & $\begin{array}{l}\text { Infection of throat and } \\
\text { flu }\end{array}$ & root, rhizome & Syrup \\
\hline Persea americana & $\begin{array}{l}\text { Cholesterol, kidney } \\
\text { stones, kidney infection }\end{array}$ & $\begin{array}{l}\text { leaves, fruits, } \\
\text { root, whole plant }\end{array}$ & Food, infusion \\
\hline Petiveria alliacea & $\begin{array}{l}\text { Cough and flu, } \\
\text { analgesic, body aches }\end{array}$ & leaves, root & Infusion \\
\hline Petroselinum crispum & Uterus infection & root, whole plant & Tea \\
\hline $\begin{array}{l}\text { Philodendron } \\
\text { bipinnatifidum }\end{array}$ & Back pain, rheumatism & leaves, fruits & Infusion in alcohol, cream \\
\hline Phyllanthus niruri & $\begin{array}{l}\text { Infections and urinary } \\
\text { problems, stomach } \\
\text { disorders } \\
\end{array}$ & whole plant & Infusion \\
\hline Piper aduncum & Wounds, healing, spice & leaves, fruits & Infusion \\
\hline Piper nigrum & $\begin{array}{l}\text { In the treatment of } \\
\text { inflammation, sinusitis } \\
\text { and digestion problems }\end{array}$ & fruits & Spice \\
\hline Plantago major & Throat & leaves & Infusion \\
\hline Plathymenia reticulata & $\begin{array}{l}\text { Gastritis, ulcer, stomach } \\
\text { infections }\end{array}$ & bast & Infusion, pill \\
\hline Plectranthus amboinicus & $\begin{array}{l}\text { Soothing, colds, cough, } \\
\text { fungicide }\end{array}$ & leaves & Infusion, maceration, syrup \\
\hline Plectranthus barbatus & $\begin{array}{l}\text { Digestive problems, } \\
\text { labyrinthitis }\end{array}$ & leaves & $\begin{array}{l}\text { Infusion or by maceration, } \\
\text { compressed powder }\end{array}$ \\
\hline Polygala longicaulis & Cold, flu & root & Tea \\
\hline Pouteria caimito & Diabetes & fruits & Food \\
\hline Prunus domestica & Cough, high pressure & fruits & Syrup \\
\hline Psidium guajava & $\begin{array}{l}\text { Toothache, acne, hair } \\
\text { loss, throat infection, } \\
\text { diarrhea }\end{array}$ & leaves, bud & Infusion, soap \\
\hline
\end{tabular}




\begin{tabular}{|c|c|c|c|}
\hline Pterodon emarginatus & $\begin{array}{l}\text { Indigestion, throat } \\
\text { infections }\end{array}$ & bark, seeds & Maceration \\
\hline Punica granatum & Throat infection & $\begin{array}{l}\text { seeds, fruit and } \\
\text { trunk bark, root } \\
\text { bark }\end{array}$ & $\begin{array}{l}\text { Decoction of } 1 / 4 \text { of the peel of a fruit } \\
\text { for } 10 \text { minutes in a glass of water }\end{array}$ \\
\hline Qualea grandiflora & $\begin{array}{l}\text { Intestinal and liver } \\
\text { problems }\end{array}$ & leaves, bast & Infusion \\
\hline Qualea parviflora & Bronchitis, cough & seeds & Infusion \\
\hline Ricinus communis & Emollient and healing & oil & Skin \\
\hline Rosa alba & $\begin{array}{l}\text { In the treatment of boils, } \\
\text { skin infections }\end{array}$ & flowers & Boiling in milk \\
\hline Rosmarinus officinalis & $\begin{array}{l}\text { Soothing, chest and heart } \\
\text { pain }\end{array}$ & leaves & Infusion \\
\hline $\begin{array}{l}\text { Roupala montana var. } \\
\text { brasiliensis }\end{array}$ & $\begin{array}{l}\text { Diarrhea, rheumatism, } \\
\text { anti-inflammatory, blood } \\
\text { purifier }\end{array}$ & leaves, bast & Infusion \\
\hline Rudgea viburnoides & $\begin{array}{l}\text { Prevention of stretch } \\
\text { marks, high blood } \\
\text { pressure, arterial } \\
\text { problems }\end{array}$ & leaves & Infusion, pill \\
\hline Rumex acetosa & Diarrhea & leaves & Infusion \\
\hline Ruta graveolens & $\begin{array}{l}\text { In stye treatment, } \\
\text { delayed menstruation }\end{array}$ & leaves & Infusion by alcohol \\
\hline Sambucus australis & $\begin{array}{l}\text { Colds, sinusitis, catarrh } \\
\text { elimination, measles and } \\
\text { arthritis }\end{array}$ & flowers and bast & Infusion \\
\hline Sedum dendroideum & $\begin{array}{l}\text { Ear pain, healing, natural } \\
\text { throat infection }\end{array}$ & leaves & Maceration, Infusion \\
\hline Senna occidentalis & Pneumonia, flu & root & Tea \\
\hline Siparuna guianensis & $\begin{array}{l}\text { erysipelas, skin } \\
\text { problems }\end{array}$ & leaves & Bath, cream, soap, infusion \\
\hline Smilax brasiliensis & Infection, snakebite & root, juice & Cream, infusion \\
\hline Solanum cernuum & Infection, post surgery & leaves & Infusion \\
\hline Solanum lycocarpum & $\begin{array}{l}\text { Diabetes, cholesterol, } \\
\text { lung infection, } \\
\text { expectorant, bronchitis, } \\
\text { hepatitis pneumonia, } \\
\text { gastritis and ulcers, skin } \\
\text { cancer }\end{array}$ & flowers, fruits & Pill, cream, shampoo, soap \\
\hline Solanum paniculatum & $\begin{array}{l}\text { Liver problems, } \\
\text { Digestive problems, flu }\end{array}$ & root, fruto & Tea, food \\
\hline Solidago chilensis & $\begin{array}{l}\text { Bruises, relieve swelling } \\
\text { and pain in the legs, as } \\
\text { repellent and anti- } \\
\text { inflammatory }\end{array}$ & whole plant & Infusion in alcohol, soap, cream, gel \\
\hline Strychnos pseudoquina & $\begin{array}{l}\text { In the treatment of } \\
\text { urinary infections, liver } \\
\text { and stomach problems }\end{array}$ & leaves, bast, root & Infusion \\
\hline
\end{tabular}

Boletín Latinoamericano y del Caribe de Plantas Medicinales y Aromáticas / 506 


\begin{tabular}{|c|c|c|c|}
\hline & and diabetes & & \\
\hline $\begin{array}{l}\text { Stryphnodendron } \\
\text { adstringens }\end{array}$ & $\begin{array}{l}\text { Healing of skin wounds, } \\
\text { gastritis, ulcer, infection } \\
\text { in the uterus and vaginal } \\
\text { discharge, leukorrhea, } \\
\text { bactericide and fungicide } \\
\end{array}$ & bast, leaves & Tea \\
\hline Symphytum officinale & $\begin{array}{l}\text { Infection, contusion, } \\
\text { baldness }\end{array}$ & leaves & Plaster \\
\hline Tagetes patula & Joint pain, rheumatism & leaves, flowers & Tea \\
\hline Tanacetum parthenium & $\begin{array}{l}\text { Helps purify the body in } \\
\text { the postpartum period } \\
\text { and regulate } \\
\text { menstruation }\end{array}$ & leaves & Tea \\
\hline Terminalia argentea & $\begin{array}{l}\text { Flu and breathing } \\
\text { problems }\end{array}$ & bast & Tea \\
\hline Tetradenia riparia & $\begin{array}{l}\text { Burns, antibacterial, } \\
\text { antifungal }\end{array}$ & leaves & Maceration \\
\hline Thevetia peruviana & $\begin{array}{l}\text { Dandruff, seborrhea, } \\
\text { cosmetics }\end{array}$ & fruits & Cosmetics \\
\hline Vanilla planifolia & Gastritis, ulcers & fruits & Maceration \\
\hline Vernonanthura polyanthes & Flu, Asthma, Bronchitis & leaves, root & Infusion, syrup \\
\hline Vochysia elliptica & $\begin{array}{l}\text { Heart problems, High } \\
\text { blood pressure, poor } \\
\text { circulation, varicose } \\
\text { veins, gastritis, Ulcers, } \\
\text { diabetes, Bronchitiss and } \\
\text { pneumonia }\end{array}$ & bark, bast & Pill, infusion \\
\hline Xanthosoma sagittifolium & Ulcer, bone pain & leaves & Sprinkle, food \\
\hline Xylopia aromatica & $\begin{array}{l}\text { Indigestion, gases, } \\
\text { constipation, sexual } \\
\text { impotence }\end{array}$ & fruits, seeds, bast & Infusion, maceration and plaster tea \\
\hline Zeyheria montana & Infections & $\begin{array}{l}\text { leaves, whole } \\
\text { plant }\end{array}$ & Infusion \\
\hline Zingiber officinale & $\begin{array}{l}\text { Thermogenic, } \\
\text { accelerates metabolism }\end{array}$ & rhizomes & Tea, powder, food \\
\hline
\end{tabular}

The guidelines for collecting the plants made by some of the interviewees were for this to occur at the time of preparation of the medicine, being on the side where the sun rises and before $10 \mathrm{am}$ and, in the specific case of collecting the bark, this must be done from a meter high in the soil so as not to harm the plant, there is no specific period or time for collection.

\section{Boletín Latinoamericano y del Caribe de Plantas Medicinales y Aromáticas / 507}




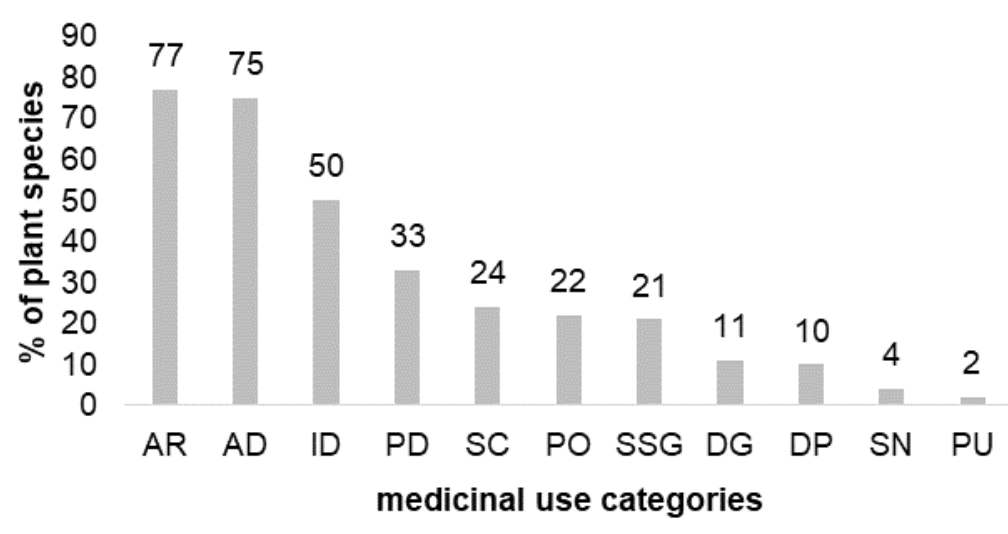

Figure No. 4

Percentage of medicinal plant species used in the Boa Esperança Settlement of Piracanjuba, GO, according to the categories of medicinal uses

The main form of preparation used with medicinal plants is tea $(55.42 \%$ of the species mentioned are consumed in this way) (Table No. 2). The use of tea as the main form of preparation of home remedy has been observed in other ethnobotanical studies (Silva et al., 2012; Costa \& Marinho, 2016; Alves et al., 2018; Guimarães et al.,
2019). According to the interviewees, teas can be prepared by infusion or decoction, depending on the part of the plant to be used. For them, infusion is recommended when using plant parts such as leaves, flowers, inflorescences and fruits, it is important not to boil the plant. Decoction is used for the hardest parts of plants, such as bark, roots, seeds and stems.

Table No 3

Percentage of agreement regarding the main use (s) of the species mentioned by five or more informants. ICUS - number of informants who cited the use of the species; ICMU - number of informants who cited main use; PMU - usage agreement index; CF - correction factor; PMUc - Corrected PMU, at the Boa Esperança Settlement, Piracanjuba, GO

\begin{tabular}{llrrrrr}
\hline \multicolumn{1}{c}{ Specie } & \multicolumn{1}{c}{ Main Use } & ICUS & ICMU & PMU & CF & PMUc \\
\hline Dilodendron bipinnatum & Spine & 6 & 6 & $\mathbf{1 0 0}$ & 1 & $\mathbf{1 0 0}$ \\
Morus sp. & Natural hormone & 6 & 6 & $\mathbf{1 0 0}$ & 1 & $\mathbf{1 0 0}$ \\
Pterodon emarginatus & Sore throat & 6 & 6 & $\mathbf{1 0 0}$ & 1 & $\mathbf{1 0 0}$ \\
Qualea grandiflora & Liver problems & 6 & 6 & $\mathbf{1 0 0}$ & 1 & $\mathbf{1 0 0}$ \\
Aloe vera & Gastritis & 6 & 5 & 83,33 & 1 & 83,33 \\
Cochlospermum regium & Infection & 5 & 5 & $\mathbf{1 0 0}$ & 0,83 & 83,33 \\
Copaifera langsdorffii & Bronchitis & 6 & 5 & 83,33 & 1 & 83,33 \\
Croton antisyphiliticus & Female infection & 5 & 5 & $\mathbf{1 0 0}$ & 0,83 & 83,33 \\
Cymbopogon citratus & Soothing & 6 & 5 & 83,33 & 1 & 83,33 \\
Dipteryx alata & Kidneys & 5 & 5 & 100 & 0,83 & 83,33 \\
Hymenaea courbaril & Ulcer & 5 & 4 & 80 & 0,83 & 66,66 \\
Stryphnodendron adstringens & Healing & 5 & 4 & 80 & 0,83 & 66,66 \\
\hline
\end{tabular}

Boletín Latinoamericano y del Caribe de Plantas Medicinales y Aromáticas / 508 
The species cited by all respondents were Morus sp., Cochlospermum regium, Aloe vera, Stryphnodendron adstringens, Dipteryx alata, Cymbopogon citratus, Copaifera langsdorffii, Croton antisyphiliticus, Pterodon emarginatus, Dilodendron bipinnatum, Qualea grandiflora and Hymenaea courbaril (Table No. 3). Most of these species are native, with Aloe vera exotic and Cymbopogon citratus naturalized. The species that obtained the maximum use agreement value were Morus sp., Dilodendron bipinnatum, Pterodon emarginatus and Qualea grandiflora.

The diversity of medicinal species used by the interviewees is high $\left(\mathrm{H}^{\prime}=4.84\right)$, as well as the
Pielou equitability index $\left(\mathrm{J}^{\prime}=0.94\right)$. These results are similar to the values found by other studies in Cerrado areas (Table No. 4). The high diversity index suggests that the settlement's residents have significant ethnobotanical knowledge when using a large number of species from the local biodiversity, in addition to species from other areas. In addition, the greater the local floristic diversity, the greater the availability of species to be used (Begossi, 1996). This is also corroborated by the high level of fairness, which indicates that knowledge about the uses of medicinal plants has a relatively uniform distribution among the interviewees (Amorozo, 2002; Cunha \& Bortolotto, 2011).

Table No 4

Comparison between the Shannon-Wiener $\left(\mathrm{H}^{\prime}\right)$ and Pielou $\left(\mathrm{J}^{\prime}\right)$ diversity indexes between ethnobotanical studies from different sites in the Cerrado phytogeographic domain. NI = number of informants; $\mathrm{NC}=$ number of citations; $\mathrm{NS}=$ number of species

\begin{tabular}{lcrrrrc}
\hline \multicolumn{1}{c}{ Place } & H' & \multicolumn{1}{c}{ J' } & \multicolumn{1}{c}{ NI } & \multicolumn{1}{c}{ NC } & \multicolumn{1}{c}{ NS } & Authors \\
\hline Boa Esperança - Piracanjuba, GO. & 4,84 & 0,94 & 12 & 398 & 178 & This manuscript \\
Monjolinho - Anastácio, MS & 5,03 & 0,94 & 35 & - & 209 & Cunha \& Bortolotto, 2011 \\
Furna São José - Cacéres, MT & 3,28 & 0,87 & 34 & - & 62 & \\
Corixo- Cacéres, MT & 2,91 & 0,82 & 29 & - & 57 & Mendes et al. (2012) \\
\hline
\end{tabular}

Jaccard's similarity indexes show little or no floristic similarity between this study and other ethnobotanical research carried out in settlements and rural communities (Table No. 5; Figure No. 5). It can be inferred that the low similarity is related to the great diversity of native and cultivated species that are intrinsic to each region of the Cerrado phytogeographic domain.

Table No. 5

Jaccard similarity index (JS) between settlements and rural communities in the Cerrado area. $A=$ this work, B = Monjolinho, MS, (Cunha \& Bortolotto, 2011), C = Vale Verde, TO (Bessa et al., 2013), $\mathrm{D}=$ Santa Rita, MG (Alves \& Povh, 2013) and E = Conceição-Açu, MT (Pasa et al., 2005)

\begin{tabular}{crrrrr}
\hline Communities & \multicolumn{1}{c}{ A } & \multicolumn{1}{c}{ B } & \multicolumn{1}{c}{ C } & \multicolumn{1}{c}{ D } & \multicolumn{1}{c}{$\mathbf{E}$} \\
\hline $\mathbf{A}$ & 1 & 0,22 & 0,03 & 0,06 & 0,24 \\
$\mathbf{B}$ & 0,22 & 1 & 0,04 & 0,04 & 0,21 \\
$\mathbf{C}$ & 0,03 & 0,01 & 1 & 0 & 0,02 \\
$\mathbf{D}$ & 0,06 & 0,04 & 0 & 1 & 0,09 \\
$\mathbf{E}$ & 0,24 & 0,21 & 0,02 & 0,09 & 1 \\
\hline
\end{tabular}

Boletín Latinoamericano y del Caribe de Plantas Medicinales y Aromáticas / 509 


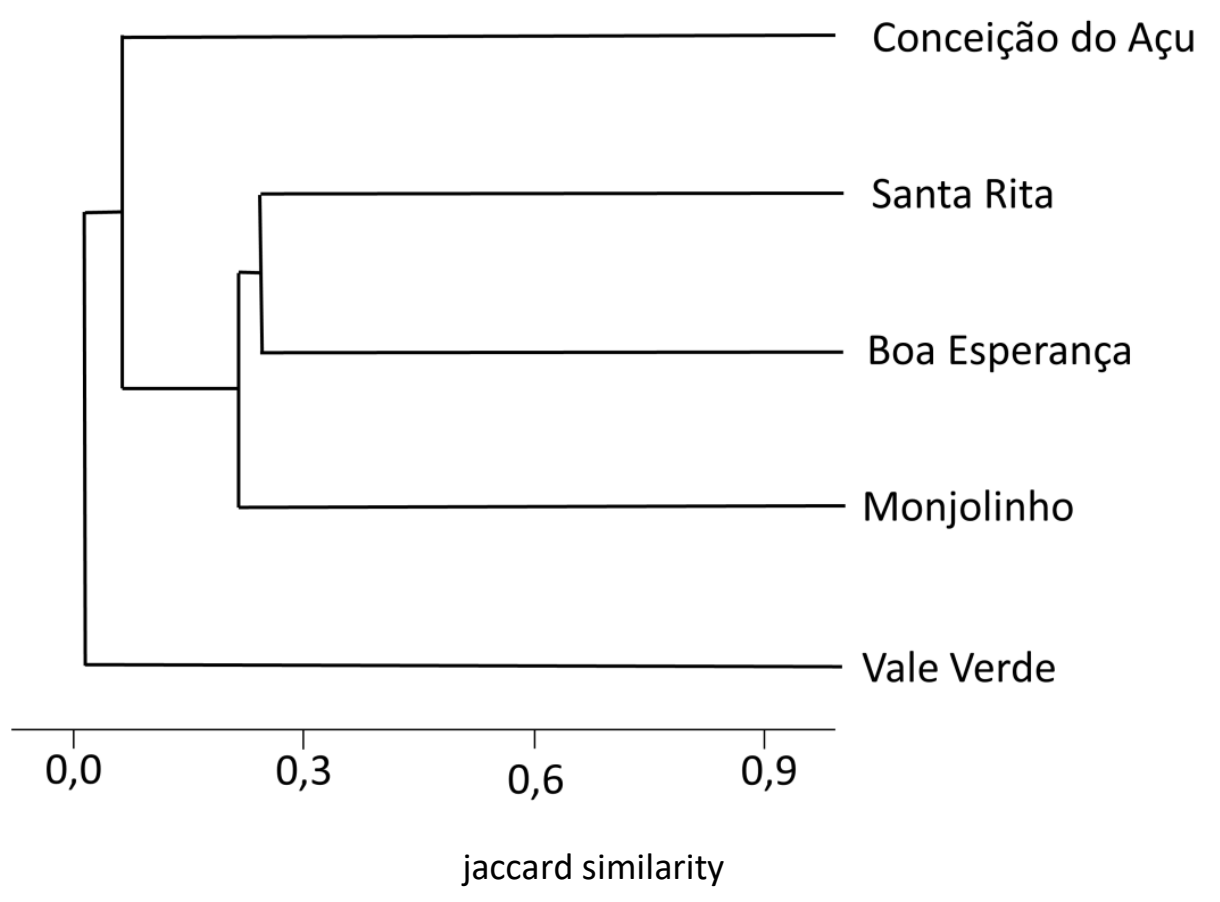

Figure No. 5

Dendrogram generated from the Jaccard similarity index (JS) between the work carried out in settlements and rural communities and the present work. $A=$ this work, $B=$ Monjolinho,

MS (Cunha \& Bortolotto, 2011), C = Vale Verde, TO (Bessa et al., 2013), D = Santa Rita, MG (Alves \& Povh, 2013) and E = Conceição -Açu, MT (Pasa et al., 2005)

\section{CONCLUSIONS}

The diversity of medicinal species recorded in the present study shows the importance and frequent use of ethnobotanical knowledge by the studied community. The low or no floristic similarity between this study and other ethnobotanical research carried out in settlements and rural communities corroborates the occurrence of a high diversity of native and cultivated species, which are intrinsic to each region of the Cerrado phytogeographic domain. However, further studies are needed to prove whether this pattern persists in space and time among traditional Cerrado communities. As for the threat status, the fact that most of the aforementioned native medicinal species have not been assessed is worrying. Therefore, it is important to expand studies to assess the threat status of these species in order to establish future conservation strategies.

\section{ACKNOWLEDGMENTS}

To the people of Boa Esperança Settlement, Piracanjuba, GO, for their valuable collaboration. To the reviewers whose suggestions made it possible to improve the quality of this research. To J Francisco Morales for his help with editing Figures No. 1 and No. 2.

\section{REFERENCES}

Albuquerque EM. 2009. Avaliação da técnica de amostragem "Respondent-driven Sampling" na estimação de prevalências de Doenças Transmissíveis em populações organizadas em redes complexas. Tesis, Escola Nacional de Saúde Pública Sérgio Arouca - ENSP. Rio de Janeiro, Brasil.

Almeida SP, Proença CEB, Sano SM, Ribeiro JF. 1998. Cerrado, Espécies vegetais úteis. EMBRAPA/CPAC, Planaltina, Brasil.

Alves GSP, Povh JA. 2013. Estudo etnobotânico de plantas medicinais na comunidade de Santa Rita. Rev Biotemas 26: 232 - 242. https://doi.org/10.5007/2175-7925.2013v26n3p231

Boletín Latinoamericano y del Caribe de Plantas Medicinales y Aromáticas / 510 
Alves HKDR, Morais IL, Caes AL. 2018. Medicina popular no Cerrado e plantas medicinais usadas pelas irmãs raízeiras de Morrinhos, GO. In: Santos FR (Org.) Economia, Política e Sociedade: vicissitudes e perspectivas para a preservação do meio ambiente no Brasil. Editora CRV, Curitiba, Brasil.

https://doi.org/10.24824/978854442140.6

Amorozo MCM, Gély AL. 1988. Uso de plantas medicinais por caboclos do baixo Amazonas, Barcarena, PA, Brasil. Bol Museu Paraense Emílio Goeldi, Série Botânica 4: 47 - 131. https://doi.org/10.1590/1981.81222019000200015

Amorozo MCM. 2002. Uso e diversidade de plantas medicinais em Santo Antônio do Leverger, MT, Brasil. Acta Bot Bras 16: 189 - 203. https://doi.org/10.1590/s0102-33062002000200006

Begossi A. 1996. Use of ecological methods in ethnobotany: Diversity indices. Econ Bot 50: 280 - 289. https://doi.org/10.1007/bf02907333

Bessa NGF de, Borges JCM, Bezerra FP, Carvalho RHA, Pereira MAB, Fagundes R, Campos SL, Ribeiro LU, Quirino MS, Chagas Junior AF, Alves A. 2013. Prospecção fitoquímica preliminar de plantas nativas do Cerrado de uso popular e medicinal pela comunidade rural do Assentamento Vale Verde - Tocantins. Rev Bras Plantas Med 15: 692 - 707. https://doi.org/10.1590/s1516-05722013000500010

Bicalho PSS, Miranda SC. 2015. Biodiversidade do Cerrado: sustentabilidade e saberes Indígenas. Élisée, Revista de Geografia da UEG 4: 53 - 67.

Borba AM, Macedo M. 2006. Plantas medicinais usadas para a saúde bucal pela comunidade do bairro Santa Cruz, Chapada dos Guimarães, MT, Brasil. Acta Bot Bras 20: 771 - 782. https://doi.org/10.1590/s0102-33062006000400003

Brito MFM, Marín EA, Cruz DD. 2017. Plantas medicinais nos assentamentos rurais em uma área de proteção no litoral do nordeste brasileiro. Ambiente \& Sociedade 20: 83 - 104.

Camargo FF, Souza TR, Costa RB. 2014. Etnoecologia e etnobotânica em ambientes de Cerrado no Estado de Mato Grosso. Interações 15: 353 - 360. https://doi.org/10.1590/s1518-70122014000200013

CNS. Ministério da Saúde. Resolução No 446. 2012. Aprova as normas regulamentadoras de pesquisas envolvendo seres humanos. Brasília, Brasil.

CNS (Conselho Nacional de Saúde). 2016. Resolução No 510, de 07 de abril de 2016. http://conselho.saude.gov.br/resolucoes/2016/Reso510.pdf

Costa JC, Marinho MGV. 2016. Etnobotânica de plantas medicinais em duas comunidades do município de Picuí, Paraíba, Brasil. Rev Bras Plant Med 18: 125 - 134. https://doi.org/10.1590/1983-084x/15_071

Cunha AS, Bortolotto IM. 2011. Etnobotânica de plantas medicinais no assentamento Monjolinho, município de Anastácio, Mato Grosso do Sul, Brasil. Acta Bot Bras 25: 685 - 698.

https://doi.org/10.1590/s0102-33062011000300022

Dias JE, Laureano LC. 2009. Farmacopéia Popular do Cerrado. Articulação Pacari (Associação Pacari), Goiás, Brasil.

Duarte GSD, Pasa MC. 2016. Agrobiodiversidade e a etnobotânica na comunidade São Benedito, Poconé, Mato Grosso, Brasil. Interações 17: 247 - 256. https://doi.org/10.20435/1984042x2016208

Dutra RMS, Souza MMO. 2017. Cerrado, revolução verde e evolução do consumo de agrotóxicos. Sociedade \& Natureza 29: 469 - 484. https://doi.org/10.14393/sn-v29n3-2017-8

Ferreira ALS, Batista CAS, Pasa MC. 2015. Uso de plantas medicinais na comunidade quilombola Mata Cavalo em Nossa Senhora do Livramento - MT, Brasil. Biodiversidade 14: 151 - 160.

Flora do Brasil 2020. Jardim Botânico do Rio de Janeiro, Río de Janeiro, Brasil. http://floradobrasil.jbrj.gov.br

Friedman J, Yaniv Z, Dafni A, Palewitch D. 1986. A preliminary classification of the healing potential of medicinal plants, based on a rational analysis of an ethnopharmacological field survey among bedouins in the negev desert, Israel. J Ethnopharmacol 16: 275 - 287. https://doi.org/10.1016/0378-8741(86)90094-2

Gois MAF, Lucas FCA, Costa JCM, Moura PHB de, Lobato GJM. 2016. Etnobotânica de espécies vegetais medicinais no tratamento de transtornos do sistema gastrointestinal. Rev Bras Plantas Med 18: 547 - 557. https://doi.org/10.1590/1983-084x/15_170

Gomes NS, Lima JPS. 2017. Uso e comercialização de plantas medicinais em Humaitá, Amazonas. Rev Bras Agroecol 2: 19 - 31.

Boletín Latinoamericano y del Caribe de Plantas Medicinales y Aromáticas / 511 
Guarim Neto G, Morais RG. de. 2003. Recursos medicinais de espécies do Cerrado de Mato Grosso:um estudo bibliográfico. Acta Bot Bras 17: 561 - 584. https://doi.org/10.1590/s0102-33062003000400009

Guimarães BO, Oliveira AP de, Morais IL. 2019. Plantas medicinais de uso popular na Comunidade Quilombola de Piracanjuba - Ana Laura, Piracanjuba, GO. Fronteiras 8: 196 - 220. https://doi.org/10.21664/2238-8869.2019v8i3.p196-220

INCRA 2017 [Instituto Nacional de Colonização e Reforma Agrária]. Informações gerais sobre os assentamentos da Reforma Agrária. http://painel.incra.gov.br/sistemas/index.php

IUCN 2016. The IUCN Red List of Threatened Species. Version 2016-2. http://www.iucnredlist.org

Krebs CJ. 1989. Ecological methodology. NY Harper and Row Publishers Inc., New York, USA.

Leandro YAS, Jardim IN, Gavilanes ML. 2017. Uso de plantas medicinais nos cuidados de saúde dos moradores de assentamento no município de Anapu, Pará, Brasil. Biodiversidade 16: 30 - 44.

Löbler L, Santos D, Rodrigues ES, Santos NRZ dos. 2014. Levantamento etnobotânico de plantas medicinais no bairro Três de Outubro da cidade de São Gabriel, RS, Brasil. Rev Bras Biociências 12: 81 - 89.

Maciel MAM, Pinto AC, Veiga Junior VF, Grynberg NF, Echevarria A. 2002. Plantas medicinais: a necessidade de estudos multidisciplinares. Quím Nova 25: 429 - 438. https://doi.org/10.1590/s0100-40422002000300016

Magurran AE. 1988. Ecological Diversity and its measurement. Princeton, New Jersey, USA.

Martin GF. 1995. Ethonobotany, a methods manual.WWF Internacional, Unesco, Royal Botanical, Londres, UK.

Medeiros MFT, Fonseca VS, Andreata RHP. 2004. Plantas medicinais e seus usos pelos sitiantes da Reserva Rio das Pedras, Mangaratiba, RJ, Brasil. Acta Bot Bras 18: 391 - 399. https://doi.org/10.1590/s0102-33062004000200019

Mendes MF, Neves SMAS, Ikeda SK, Castrillon SKI, Silva JSV, Neves RJ, Paiva SLP, Pedroga JA. 2012. Diversidade e distribuição espacial de espécies nativas arbóreas do Cerrado em áreas de assentamentos rurais na região sudoeste mato-grossense, Brasil. Bonito, MS. In: $4^{\circ}$ Simpósio de Geotecnologias no Pantanal. Embrapa Informática Agropecuária 1: 806 - 818.

Mendonça RC. 1998. Flora vascular do Cerrado. In: Sano SM, Almeida SP. Cerrado: Ambiente e flora. EMBRAPA - CPAC, Planaltina, Brazil.

Mobot (Missouri Botanical Garden). 2018. https://www.tropicos.org

Mota LLS, Rodrigues MM, Jones KM, Lacerda GA 2015. Abordagem etnobotânica continuada na Comunidade Remanescente Quilombola Palmeirinha, Pedras de Maria da Cruz - MG. Revista Cerrados 13: 156 - 172.

Oliveira FCS, Barros RFM, Moita Neto, JM. 2010. Plantas medicinais utilizadas em comunidades rurais de Oeiras, Semiárido Piauiense. Rev Bras Plant Med13: 82 - 292. https://doi.org/10.1590/s1516-05722010000300006

Oliveira ER, Menini Neto L. 2012. Levantamento etnobotânico de plantas medicinais utilizadas pelos moradores do povoado de Manejo, Lima Duarte - MG. Rev Bras Plant Med 14: 311 - 320. https://doi.org/10.1590/s1516-05722012000200010

Pasa MC, Soares JJ, Guarim Neto G. 2005. Estudo etnobotânico na comunidade de Conceição-Açu (Alto da Bacia do Rio Aricá Açu, MT, Brasil). Acta Bot Bras 19: 195 - 207.

https://doi.org/10.1590/s0102-33062005000200001

Pasa MC, David M, Fiebig GA, Nardez TMB, Maziero ELA. 2015. Etnobotânica na comunidade quilombola em Nossa Senhora do Livramento, Mato Grosso, Brasil. Biodiversidade 14: 2 - 18.

Pilla MAC, Amorozo MCM, Furlan A. 2006. Obtenção e uso das plantas medicinais no distrito de Martim Francisco, Município de Mogi-Mirim, SP, Brasil. Acta Bot Bras 20: 789 - 802.

https://doi.org/10.1590/s0102-33062006000400005

Pitta FT, Vega GC. 2017. Impactos da expansão do agronegócio no MATOPIBA: comunidades e meio ambiente. ActionAid, Rio de Janeiro, Brazil.

Pielou EC. 1966. The measurement of diversity in different types of biological collections. J Theor Biol 13: 131 144. https://doi.org/10.1016/0022-5193(66)90013-0

Pinto EPP, Amorozo MCM, Furlan A. 2006. Conhecimento popular sobre plantas medicinais em comunidades rurais de Mata Atlântica - Itacaré, BA, Brasil. Acta Bot Bras 20: 751 - 762.

https://doi.org/10.1590/s0102-33062006000400001

Boletín Latinoamericano y del Caribe de Plantas Medicinales y Aromáticas / 512 
Porto-Gonçalves CW. 2019. Dos Cerrados e de suas riquezas: de saberes vernaculares e de conhecimento científico. FASE e CPT, Rio de Janeiro e Goiânia, Brazil.

Rodrigues AP, Andrade LHC. 2014. Levantamento etnobotânico das plantas medicinais utilizadas pela comunidade de Inhamã, Pernambuco, Nordeste do Brasil. Rev Bras Plantas Med 16: 721 - 730. https://doi.org/10.1590/1983-084x/08_159

Silva CSP, Proença CEB. 2007. Flora medicinal nativa do bioma Cerrado catalogada por estudos etnobotânicos no estado de Goiás, Brasil. Revista Anhangüera 8: 67 - 88.

Silva JS, Carvalho JNF, Teixeira WS, Franco IO, Ribeiro DD. 2010. Importância do uso de plantas medicinais em comunidades rurais no sudoeste de Goiás. Cadernos de Agroecologia 5: 1 - 4.

Silva NCB, Regis ACD, Almeida MZ. 2012. Estudo etnobotânico em comunidades remanescentes de Quilombo em Rio de Contas-Chapada Diamantina-BA. Rev Fitos 7: 99 - 109.

Silva RSG, Peixoto JC. 2013. Acanthaceae do bioma Cerrado: identificação dos fitoquímicos das folhas da espécie Justicia thunbergioides (Lindau) Leonard (Acanthaceae) ocorrente no Parque Estadual Serra dos Pireneus, Pirenópolis, GO. Fronteiras 2: 16 - 27. https://doi.org/10.21664/2238-8869.2013v2i1.p16-27

Vásquez SPF, Mendonça MS, Noda SN. 2014. Etnobotânica de plantas medicinais em comunidades ribeirinhas do Município de Manacapuru, Amazonas, Brasil. Acta Amazonica 44: 457 - 472.

https://doi.org/10.1590/1809-4392201400423

Boletín Latinoamericano y del Caribe de Plantas Medicinales y Aromáticas / 513 